\title{
Budget outturns of Groatian municipalities, cities and counties for 2014
}

\author{
KATARINA OTT, Institute of Public Finance \\ MIHAELA BRONIĆ, Institute of Public Finance
}

\begin{abstract}
This paper seeks to clearly present the basic data on the budget outturns of Croatian local government units ${ }^{1}$ for 20I4. It provides synthesized tables based on the Ministry of Finance's database and includes revenues and expenditures ${ }^{2}$, as well as surpluses or deficits (in per capita and total terms). Also presented are data on the population ${ }^{3}$ and the levels of budget transparency of all the local government units. This paper and the tables additionally provided in the Excel format allow readers to understand the financial condition of their respective local government units, and it could also serve as a basis for an analysis necessary for taking decisions on a potential territorial and fiscal reorganisation of the country.
\end{abstract}

When analysing the budget transparency of Croatian local government units in the period between November 2014 and March 2015, huge effort was needed to collect basic data on the local government units' budgets ${ }^{4}$. Although the Ministry of Finance publishes the budget outturns of all local government units $^{5}$, this is done in the form of rather oversized Excel tables, with the cities and municipalities arranged by county. Given the large number of local government units (whose complete budgets are presented), it is often difficult to navigate through these tables. This article gives the reader a snapshot of the basic financial conditions of the local government units in 20I4, while more detailed information can be found on the website of the Ministry of Finance and the relevant local government unit. However, some notes may still be necessary for a proper understanding of the presented data.

First, the amounts for counties, cities and municipalities should not be compared, because each of these types of local government units has the authority to collect different types of revenues and provide different kinds of public goods and services. This fact is reflected in, for example, different amounts of average per capita expenditures of counties (HRK I,O70), municipalities (HRK 3,082) and cities (including

\footnotetext{
I For the purposes of this article, the term "local government units" refers to all the counties, cities and municipalities, and the term "local budgets" includes the budgets of all the counties, cities and municipalities.

${ }^{2}$ Receipts from financial assets and borrowing, and outlays on financial assets and loan repayment are excluded.

${ }^{3}$ According to the CBS (2015).

4 The results of the analysis are available in Ott, Bronić and Petrušić (2015) and at: http:/ /www.ijf.hr/transparency.

5 The Ministry of Finance's website (2015) offers the data for 1998-20I4.
} 
the City of Zagreb - HRK 3.999 ${ }^{6}$. Any person can add up the per capita expenditures of their respective municipality or city and county, in order to establish the amount of total per capita local spending at the level of that municipality or city. The City of Zagreb is an exception, as it is both a city and a county. Therefore, the citizens of Zagreb need to consider only the Zagreb data.

Secondly, it should be borne in mind that the local government units budget does not necessarily reflect the actual financial condition of a local government unit, because local government units often take out loans through companies or institutions in their (co-)ownership, rather than through their budgets? In order to establish the actual financial condition of a local government unit, one should have access not only to the local government unit's budget data, but also data on the revenues/expenditures of the companies and institutions in its (co-)ownership (e.g. utility companies). However, the data on such companies or institutions are not publicly available in one place.

Thirdly, major differences between revenues and expenditures in a given year may not be an indicator of bad local budget planning or poor outturns, but rather the consequence of large asset sales (e.g. the sales of land or buildings) or large-scale investments (e.g. in local infrastructure). Part of the data for each of the local government units can be found on the Ministry of Finance's website. However, for a full picture of a local government unit's financial condition, the Ministry should, in addition to the data on the revenue and expenditure outturns, also publish annual figures from the balance sheets of all the local government units, i.e. the data on their assets, liabilities and capital. ${ }^{8}$

Although this article is not aimed at analysing the financial condition of local government units in 2OI4, but rather at providing a synthesis of data from the Ministry of Finance's database, note should be taken of some interesting facts that immediately catch the eye:

- $\quad$ All of the twenty municipalities with the highest per capita revenues, ranging from HRK 19.313 (Vir) to HRK 8.239 (Rogoznica), but one (Plitvička Jezera), are located along the Adriatic coast, while none of the twenty poorest municipalities, with the revenues below HRK I,OOO per capita, are situated along the sea-coast.

- All of the twenty cities with the highest per capita revenues, ranging from HRK II,659 (Novalja) to HRK 6,9I4 (Crikvenica), but four (Vrlika, Lipik, Pazin and Zagreb) are coastal cities; none of the poorest cities, with the revenues below HRK 2,00o per capita, are situated along the sea-coast.

- There are huge differences among municipalities in terms of total revenues which range from over HRK 6om in Konavle, Župa Dubrovačka and Matulji to HRK 700,ooo in Zažablje. As many as 20 municipalities reported total revenues below HRK $2 \mathrm{~m}$.

- Excluding the City of Zagreb with total revenues of HRK 6.7bn, the revenues of Split and Rijeka (HRK 729m and HRK 673m respectively) are a hundred times as high as those of the cities with lowest revenues, such as Hrvatska Kostajnica, Komiža and Klanjec (HRK 7m each).

- So, we have at the same time, the above-mentioned cities with total revenues of HRK 7m, and the above-mentioned municipalities with total revenues of over HRK 6om!

- 52 municipalities have a population of over 5,000, six of them having more than 10,000 inhabitants, while 60 cities have a population below I0,000, I8 of them having less than 5,000 inhabitants.

\footnotetext{
${ }^{6}$ Given the City of Zagreb's per capita expenditures of HRK 9,662, the average for cities excluding Zagreb would be lower (HRK 3,954).

7 For more information on the borrowing of companies (co-)owned by local government units, see Bajo and Primorac (2014).

${ }^{8}$ It is possible that part of these data may already, or will soon be available on the local government units' websites, because, starting this year, all local government units, budget and extra-budgetary users are obliged, pursuant to Article I2 of the Budget Act, to publish their annual financial statements on their official websites no later than eight days from their submission to the Financial Agency.
} 
- Almost all counties, except for the Sisak-Moslavina County, experienced either a slight surplus or a marginal deficit of revenues over expenditures.

- Some municipalities and cities stand out for the fact that their total expenditures markedly exceed the revenues, e.g. the municipalities of Sveti Petar u Šumi (by as much as III\%), Oprtalj (by I03\%) and Koprivnički Ivanec (by 97\%), and the cities of Belišće (by 39\%) and Skradin (by 37\%).

- A marked surplus of total revenues over expenditures was reported by the municipalities of Viljevo (by IIo\%), Tisno (by 98\%) and Stankovci (by 73\%), and the cities of Senj (by 50\%) and Zlatar (by $47 \%$ ).

In accordance with the motto "If you collect and process data, publish them; if there are no published data, demand them; if the data are published, use them!", tables are also provided in the Excel format. Those interested can download them to their own computers for the purpose of using and rearranging them.

It can be assumed that this synthesis (which is also a kind of analysis) of the rich database maintained by the Ministry of Finance will prompt readers to visit the base individually in order to get detailed information on their local government units' budgets and to participate in analysing and deciding on how their local budget funds are spent. It is also hoped that it will serve as a basis for an analysis which should precede decision-making on a potential territorial and fiscal reorganisation of the country.

Table I

Budget outturns of municipalities, 2014

Per capita (in HRK)

Total (in million HRK)

\begin{tabular}{|c|c|c|c|c|c|c|c|c|c|}
\hline & Municipality & $\begin{array}{c}\text { Reve- } \\
\text { nues }\end{array}$ & $\begin{array}{l}\text { Expen- } \\
\text { ditures }\end{array}$ & $\begin{array}{c}\text { Surplus } \\
\text { or } \\
\text { deficit }\end{array}$ & $\begin{array}{c}\text { Popula- } \\
\text { tion }\end{array}$ & $\begin{array}{c}\text { Reve- } \\
\text { nues }\end{array}$ & $\begin{array}{l}\text { Expen- } \\
\text { ditures }\end{array}$ & $\begin{array}{c}\text { Surplus } \\
\text { or } \\
\text { deficit }\end{array}$ & $\begin{array}{c}\text { Level of } \\
\text { budget } \\
\text { trans- } \\
\text { parency }\end{array}$ \\
\hline 1 & Vir & 19,313 & 13,654 & 5,659 & 3,000 & 57.9 & 41.0 & 17.0 & 3 \\
\hline 2 & Funtana & 12,871 & 19,606 & $-6,735$ & 907 & 11.7 & 17.8 & -6.1 & 3 \\
\hline 3 & Šolta & 12,856 & 12,676 & 181 & 1,700 & 21.9 & 21.5 & 0.3 & 1 \\
\hline 4 & Baška & 12,735 & 10,785 & 1,950 & 1,674 & 21.3 & 18.1 & 3.3 & 2 \\
\hline 5 & Sutivan & 12,042 & 11,133 & 909 & 822 & 9.9 & 9.2 & 0.7 & 0 \\
\hline 6 & Bol & 11,772 & 10,308 & 1,464 & 1,630 & 19.2 & 16.8 & 2.4 & 0 \\
\hline 7 & Kolan & 10,928 & 11,253 & -326 & 791 & 8.6 & 8.9 & -0.3 & 0 \\
\hline 8 & Kostrena & 10,599 & 10,295 & 305 & 4,180 & 44.3 & 43.0 & 1.3 & 0 \\
\hline 9 & Omišalj & 10,581 & 10,059 & 522 & 2,983 & 31.6 & 30.0 & 1.6 & 1 \\
\hline 10 & Tisno & 10,578 & 5,321 & 5,257 & 3,094 & 32.7 & 16.5 & 16.3 & 0 \\
\hline 11 & $\begin{array}{l}\text { Malinska- } \\
\text { Dubašnica }\end{array}$ & 10,535 & 11,456 & -920 & 3,134 & 33.0 & 35.9 & -2.9 & 3 \\
\hline 12 & Tar-Vabriga & 10,474 & 11,615 & $-1,142$ & 1,990 & 20.8 & 23.1 & -2.3 & 0 \\
\hline 13 & Bale & 9,616 & 7,947 & 1,669 & 1,127 & 10.8 & 9.0 & 1.9 & 3 \\
\hline 14 & Karlobag & 9,372 & 9,158 & 213 & 917 & 8.6 & 8.4 & 0.2 & 0 \\
\hline 15 & Punat & 9,108 & 7,456 & 1,651 & 1,973 & 18.0 & 14.7 & 3.3 & 3 \\
\hline 16 & Vrsar & 9,005 & 8,554 & 450 & 2,162 & 19.5 & 18.5 & 1.0 & 1 \\
\hline 17 & Medulin & 8,892 & 7,556 & 1,336 & 6,481 & 57.6 & 49.0 & 8.7 & 3 \\
\hline 18 & Plitvička Jezera & 8,889 & 6,349 & 2,540 & 4,373 & 38.9 & 27.8 & 11.1 & 2 \\
\hline 19 & Fažana & 8,550 & 8,316 & 234 & 3,635 & 31.1 & 30.2 & 0.9 & 4 \\
\hline 20 & Rogoznica & 8,239 & 7,545 & 694 & 2,345 & 19.3 & 17.7 & 1.6 & 1 \\
\hline 21 & Grožnjan & 8,147 & 10,592 & $-2,445$ & 736 & 6.0 & 7.8 & -1.8 & 2 \\
\hline 22 & Fužine & 8,101 & 7,178 & 923 & 1,592 & 12.9 & 11.4 & 1.5 & 0 \\
\hline 23 & Primošten & 8,096 & 7,037 & 1,059 & 2,828 & 22.9 & 19.9 & 3.0 & 2 \\
\hline 24 & Civljane & 7,952 & 7,709 & 243 & 239 & 1.9 & 1.8 & 0.1 & 3 \\
\hline 25 & Dugopolje & 7,691 & 6,751 & 940 & 3,469 & 26.7 & 23.4 & 3.3 & 0 \\
\hline 26 & Postira & 7,623 & 6,909 & 714 & 1,559 & 11.9 & 10.8 & 1.1 & 1 \\
\hline 27 & Mljet & 7,599 & 6,889 & 710 & 1,088 & 8.3 & 7.5 & 0.8 & 1 \\
\hline 28 & Župa Dubrovačka & 7,531 & 6,607 & 923 & 8,331 & 62.7 & 55.0 & 7.7 & 0 \\
\hline 29 & Zadvarje & 7,426 & 8,217 & -791 & 289 & 2.1 & 2.4 & -0.2 & 0 \\
\hline
\end{tabular}

NEWSLETTER 98 | K. OTT \& M. BRONIĆ | Budget outturns of Croatian municipalities, cities... | Institute of Public Finance 3 
Per capita (in HRK)

Total (in million HRK)

\begin{tabular}{|c|c|c|c|c|c|c|c|c|c|}
\hline & Municipality & $\begin{array}{c}\text { Reve- } \\
\text { nues }\end{array}$ & $\begin{array}{l}\text { Expen- } \\
\text { ditures }\end{array}$ & $\begin{array}{c}\text { Surplus } \\
\text { or } \\
\text { deficit }\end{array}$ & $\begin{array}{l}\text { Popula- } \\
\text { tion }\end{array}$ & $\begin{array}{c}\text { Reve- } \\
\text { nues }\end{array}$ & $\begin{array}{l}\text { Expen- } \\
\text { ditures }\end{array}$ & $\begin{array}{c}\text { Surplus } \\
\text { or } \\
\text { deficit }\end{array}$ & $\begin{array}{c}\text { Level of } \\
\text { budget } \\
\text { trans- } \\
\text { parency }\end{array}$ \\
\hline 30 & Starigrad & 7,375 & 8,084 & -710 & 1,876 & 13.8 & 15.2 & -1.3 & 3 \\
\hline 31 & Konavle & 7,348 & 8,278 & -930 & 8,577 & 63.0 & 71.0 & -8.0 & 1 \\
\hline 32 & Povljana & 7,278 & 7,062 & 216 & 759 & 5.5 & 5.4 & 0.2 & 0 \\
\hline 33 & Ravna Gora & 7,209 & 7,096 & 114 & 2,430 & 17.5 & 17.2 & 0.3 & 1 \\
\hline 34 & Milna & 7,013 & 7,939 & -926 & 1,034 & 7.3 & 8.2 & -1.0 & 1 \\
\hline 35 & Pučišća & 7,006 & 6,982 & 24 & 2,171 & 15.2 & 15.2 & 0.1 & 1 \\
\hline 36 & Janjina & 6,818 & 6,847 & -29 & 551 & 3.8 & 3.8 & 0.0 & 1 \\
\hline 37 & Lopar & 6,685 & 6,384 & 302 & 1,263 & 8.4 & 8.1 & 0.4 & 1 \\
\hline 38 & $\begin{array}{l}\text { Dubrovačko } \\
\text { Primorje }\end{array}$ & 6,655 & 8,789 & $-2,134$ & 2,170 & 14.4 & 19.1 & -4.6 & 0 \\
\hline 39 & Baška Voda & 6,546 & 5,662 & 884 & 2,775 & 18.2 & 15.7 & 2.5 & 1 \\
\hline 40 & Kršan & 6,542 & 5,987 & 555 & 2,951 & 19.3 & 17.7 & 1.6 & 1 \\
\hline 41 & Brtonigla & 6,528 & 5,429 & 1,099 & 1,626 & 10.6 & 8.8 & 1.8 & 3 \\
\hline 42 & $\begin{array}{l}\text { Mošćenička } \\
\text { Draga }\end{array}$ & 6,382 & 6,810 & -428 & 1,535 & 9.8 & 10.5 & -0.7 & 4 \\
\hline 43 & Vrbnik & 6,327 & 5,082 & 1,246 & 1,260 & 8.0 & 6.4 & 1.6 & 4 \\
\hline 44 & Pićan & 6,292 & 5,371 & 921 & 1,827 & 11.5 & 9.8 & 1.7 & 1 \\
\hline 45 & Dobrinj & 6,246 & 5,831 & 415 & 2,078 & 13.0 & 12.1 & 0.9 & 3 \\
\hline 46 & Nerežišća & 6,175 & 6,985 & -811 & 862 & 5.3 & 6.0 & -0.7 & 0 \\
\hline 47 & Lastovo & 6,140 & 5,694 & 446 & 792 & 4.9 & 4.5 & 0.4 & 3 \\
\hline 48 & Saborsko & 5,950 & 6,265 & -316 & 632 & 3.8 & 4.0 & -0.2 & 2 \\
\hline 49 & Tribunj & 5,913 & 5,861 & 52 & 1,536 & 9.1 & 9.0 & 0.1 & 3 \\
\hline 50 & Motovun & 5,835 & 5,708 & 127 & 1,004 & 5.9 & 5.7 & 0.1 & 0 \\
\hline 51 & Tkon & 5,828 & 4,919 & 909 & 763 & 4.4 & 3.8 & 0.7 & 1 \\
\hline 52 & Brela & 5,752 & 6,215 & -462 & 1,703 & 9.8 & 10.6 & -0.8 & 2 \\
\hline 53 & Kalinovac & 5,685 & 7,618 & $-1,933$ & 1,597 & 9.1 & 12.2 & -3.1 & 2 \\
\hline 54 & Sveti Lovreč & 5,680 & 5,768 & -88 & 1,015 & 5.8 & 5.9 & -0.1 & 1 \\
\hline 55 & Kijevo & 5,644 & 4,899 & 745 & 417 & 2.4 & 2.0 & 0.3 & 0 \\
\hline 56 & Jasenice & 5,618 & 6,897 & $-1,278$ & 1,398 & 7.9 & 9.6 & -1.8 & 2 \\
\hline 57 & Lovinac & 5,617 & 6,605 & -987 & 1,007 & 5.7 & 6.7 & -1.0 & 3 \\
\hline 58 & Matulji & 5,391 & 4,607 & 784 & 11,246 & 60.6 & 51.8 & 8.8 & 4 \\
\hline 59 & Vižinada & 5,367 & 3,346 & 2,021 & 1,158 & 6.2 & 3.9 & 2.3 & 1 \\
\hline 60 & Murter & 5,363 & 5,324 & 38 & 2,044 & 11.0 & 10.9 & 0.1 & 3 \\
\hline 61 & Podgora & 5,306 & 6,316 & $-1,010$ & 2,518 & 13.4 & 15.9 & -2.5 & 0 \\
\hline 62 & Lovran & 5,298 & 5,396 & -98 & 4,101 & 21.7 & 22.1 & -0.4 & 1 \\
\hline 63 & Molve & 5,265 & 5,758 & -493 & 2,189 & 11.5 & 12.6 & -1.1 & 3 \\
\hline 64 & Lećevica & 5,263 & 7,908 & $-2,644$ & 583 & 3.1 & 4.6 & -1.5 & 2 \\
\hline 65 & Kanfanar & 5,082 & 5,305 & -223 & 1,543 & 7.8 & 8.2 & -0.3 & 3 \\
\hline 66 & Tučepi & 5,065 & 5,107 & -42 & 1,931 & 9.8 & 9.9 & -0.1 & 0 \\
\hline 67 & Kukljica & 4,981 & 4,907 & 74 & 714 & 3.6 & 3.5 & 0.1 & 0 \\
\hline 68 & Ston & 4,883 & 5,176 & -293 & 2,407 & 11.8 & 12.5 & -0.7 & 3 \\
\hline 69 & Nova Bukovica & 4,876 & 5,155 & -279 & 1,771 & 8.6 & 9.1 & -0.5 & 0 \\
\hline 70 & Magadenovac & 4,844 & 4,763 & 81 & 1,936 & 9.4 & 9.2 & 0.2 & 1 \\
\hline 71 & Rakovica & 4,804 & 4,170 & 634 & 2,387 & 11.5 & 10.0 & 1.5 & 4 \\
\hline 72 & Blato & 4,794 & 4,041 & 753 & 3,593 & 17.2 & 14.5 & 2.7 & 3 \\
\hline 73 & Klana & 4,785 & 3,595 & 1,190 & 1,975 & 9.4 & 7.1 & 2.3 & 0 \\
\hline 74 & Lokve & 4,765 & 4,411 & 353 & 1,049 & 5.0 & 4.6 & 0.4 & 1 \\
\hline 75 & Gračišće & 4,704 & 2,878 & 1,825 & 1,419 & 6.7 & 4.1 & 2.6 & 3 \\
\hline 76 & Sali & 4,697 & 3,622 & 1,075 & 1,698 & 8.0 & 6.2 & 1.8 & 2 \\
\hline 77 & Okrug & 4,683 & 5,528 & -845 & 3,349 & 15.7 & 18.5 & -2.8 & 2 \\
\hline 78 & Čavle & 4,683 & 4,459 & 224 & 7,220 & 33.8 & 32.2 & 1.6 & 2 \\
\hline 79 & Tinjan & 4,673 & 2,774 & 1,899 & 1,684 & 7.9 & 4.7 & 3.2 & 0 \\
\hline 80 & Jelsa & 4,637 & 4,603 & 34 & 3,582 & 16.6 & 16.5 & 0.1 & 0 \\
\hline 81 & Stubičke Toplice & 4,602 & 4,644 & -42 & 2,805 & 12.9 & 13.0 & -0.1 & 3 \\
\hline 82 & $\begin{array}{l}\text { Vinodolska } \\
\text { Općina }\end{array}$ & 4,591 & 4,591 & -1 & 3,577 & 16.4 & 16.4 & 0.0 & 1 \\
\hline
\end{tabular}


Per capita (in HRK)

Total (in million HRK)

\begin{tabular}{|c|c|c|c|c|c|c|c|c|c|}
\hline & Municipality & $\begin{array}{c}\text { Reve- } \\
\text { nues }\end{array}$ & $\begin{array}{l}\text { Expen- } \\
\text { ditures }\end{array}$ & $\begin{array}{c}\text { Surplus } \\
\text { or } \\
\text { deficit }\end{array}$ & $\begin{array}{l}\text { Popula- } \\
\text { tion }\end{array}$ & $\begin{array}{c}\text { Reve- } \\
\text { nues }\end{array}$ & $\begin{array}{l}\text { Expen- } \\
\text { ditures }\end{array}$ & $\begin{array}{c}\text { Surplus } \\
\text { or } \\
\text { deficit }\end{array}$ & $\begin{array}{c}\text { Level of } \\
\text { budget } \\
\text { trans- } \\
\text { parency }\end{array}$ \\
\hline 83 & Stupnik & 4,568 & 3,984 & 584 & 3,735 & 17.1 & 14.9 & 2.2 & 3 \\
\hline 84 & Trpanj & 4,542 & 4,377 & 165 & 721 & 3.3 & 3.2 & 0.1 & 3 \\
\hline 85 & Raša & 4,540 & 4,415 & 125 & 3,183 & 14.5 & 14.1 & 0.4 & 1 \\
\hline 86 & Pirovac & 4,474 & 3,905 & 569 & 1,930 & 8.6 & 7.5 & 1.1 & 4 \\
\hline 87 & Pisarovina & 4,454 & 4,127 & 327 & 3,689 & 16.4 & 15.2 & 1.2 & 0 \\
\hline 88 & Mrkopalj & 4,425 & 4,362 & 63 & 1,214 & 5.4 & 5.3 & 0.1 & 2 \\
\hline 89 & Perušić & 4,415 & 3,239 & 1,175 & 2,638 & 11.6 & 8.5 & 3.1 & 1 \\
\hline 90 & Pakoštane & 4,349 & 4,676 & -328 & 4,123 & 17.9 & 19.3 & -1.4 & 0 \\
\hline 91 & Primorski Dolac & 4,270 & 6,315 & $-2,046$ & 770 & 3.3 & 4.9 & -1.6 & 2 \\
\hline 92 & Oprtalj & 4,216 & 8,569 & $-4,353$ & 850 & 3.6 & 7.3 & -3.7 & 1 \\
\hline 93 & Stankovci & 4,205 & 2,419 & 1,785 & 2,003 & 8.4 & 4.8 & 3.6 & 0 \\
\hline 94 & Lupoglav & 4,192 & 6,528 & $-2,336$ & 924 & 3.9 & 6.0 & -2.2 & 0 \\
\hline 95 & Preko & 4,177 & 4,161 & 16 & 3,805 & 15.9 & 15.8 & 0.1 & 0 \\
\hline 96 & Orebić & 4,155 & 3,933 & 221 & 4,122 & 17.1 & 16.2 & 0.9 & 0 \\
\hline 97 & Zemunik Donji & 4,064 & 5,263 & $-1,198$ & 2,060 & 8.4 & 10.8 & -2.5 & 0 \\
\hline 98 & Barilović & 4,059 & 2,493 & 1,566 & 2,990 & 12.1 & 7.5 & 4.7 & 1 \\
\hline 99 & Drenovci & 4,040 & 3,267 & 773 & 5,174 & 20.9 & 16.9 & 4.0 & 3 \\
\hline 100 & Pašman & 4,038 & 5,139 & $-1,101$ & 2,082 & 8.4 & 10.7 & -2.3 & 2 \\
\hline 101 & Crnac & 4,024 & 3,169 & 855 & 1,456 & 5.9 & 4.6 & 1.2 & 4 \\
\hline 102 & Privlaka (Zadar) & 3,900 & 3,252 & 648 & 2,253 & 8.8 & 7.3 & 1.5 & 3 \\
\hline 103 & $\begin{array}{l}\text { Podravske } \\
\text { Sesvete }\end{array}$ & 3,869 & 4,319 & -449 & 1,630 & 6.3 & 7.0 & -0.7 & 0 \\
\hline 104 & Cerovlje & 3,869 & 3,797 & 73 & 1,677 & 6.5 & 6.4 & 0.1 & 1 \\
\hline 105 & Vrsi & 3,860 & 3,720 & 140 & 2,053 & 7.9 & 7.6 & 0.3 & 0 \\
\hline 106 & Viškovo & 3,846 & 4,816 & -970 & 14,445 & 55.6 & 69.6 & -14.0 & 5 \\
\hline 107 & Sveti Filip i Jakov & 3,826 & 3,400 & 426 & 4,606 & 17.6 & 15.7 & 2.0 & 2 \\
\hline 108 & Sućuraj & 3,824 & 6,372 & $-2,548$ & 463 & 1.8 & 3.0 & -1.2 & 0 \\
\hline 109 & Kali & 3,780 & 3,661 & 119 & 1,638 & 6.2 & 6.0 & 0.2 & 1 \\
\hline 110 & Koprivnički Bregi & 3,758 & 3,752 & 6 & 2,381 & 8.9 & 8.9 & 0.0 & 3 \\
\hline 111 & Prgomet & 3,745 & 3,346 & 399 & 673 & 2.5 & 2.3 & 0.3 & 0 \\
\hline 112 & Svetvinčenat & 3,737 & 4,335 & -598 & 2,202 & 8.2 & 9.5 & -1.3 & 0 \\
\hline 113 & Jalžabet & 3,670 & 3,126 & 544 & 3,615 & 13.3 & 11.3 & 2.0 & 1 \\
\hline 114 & Novigrad & 3,669 & 4,479 & -810 & 2,375 & 8.7 & 10.6 & -1.9 & 1 \\
\hline 115 & $\begin{array}{l}\text { Kneževi } \\
\text { Vinogradi }\end{array}$ & 3,666 & 4,214 & -548 & 4,614 & 16.9 & 19.4 & -2.5 & 1 \\
\hline 116 & $\begin{array}{l}\text { Sveta Nedelja } \\
\text { (Istra) }\end{array}$ & 3,645 & 4,148 & -503 & 2,987 & 10.9 & 12.4 & -1.5 & 4 \\
\hline 117 & Legrad & 3,642 & 4,418 & -775 & 2,241 & 8.2 & 9.9 & -1.7 & 1 \\
\hline 118 & Skrad & 3,639 & 3,777 & -138 & 1,062 & 3.9 & 4.0 & -0.1 & 3 \\
\hline 119 & Marčana & 3,637 & 3,589 & 48 & 4,253 & 15.5 & 15.3 & 0.2 & 3 \\
\hline 120 & Ribnik & 3,608 & 3,435 & 173 & 475 & 1.7 & 1.6 & 0.1 & 4 \\
\hline 121 & Ljubešćica & 3,587 & 3,761 & -174 & 1,858 & 6.7 & 7.0 & -0.3 & 2 \\
\hline 122 & Jasenovac & 3,556 & 2,485 & 1,071 & 1,997 & 7.1 & 5.0 & 2.1 & 1 \\
\hline 123 & Lanišće & 3,555 & 3,802 & -247 & 329 & 1.2 & 1.3 & -0.1 & 2 \\
\hline 124 & Polača & 3,522 & 3,263 & 259 & 1,468 & 5.2 & 4.8 & 0.4 & 1 \\
\hline 125 & Klis & 3,513 & 3,786 & -272 & 4,801 & 16.9 & 18.2 & -1.3 & 2 \\
\hline 126 & Marina & 3,494 & 3,644 & -150 & 4,595 & 16.1 & 16.7 & -0.7 & 0 \\
\hline 127 & Poličnik & 3,494 & 3,465 & 29 & 4,469 & 15.6 & 15.5 & 0.1 & 0 \\
\hline 128 & Jesenje & 3,435 & 3,599 & -164 & 1,560 & 5.4 & 5.6 & -0.3 & 2 \\
\hline 129 & Kaštelir - Labinci & 3,396 & 3,975 & -579 & 1,463 & 5.0 & 5.8 & -0.8 & 1 \\
\hline 130 & Radoboj & 3,351 & 3,122 & 229 & 3,387 & 11.3 & 10.6 & 0.8 & 1 \\
\hline 131 & Hrvace & 3,336 & 3,146 & 190 & 3,617 & 12.1 & 11.4 & 0.7 & 0 \\
\hline 132 & Marija Gorica & 3,326 & 2,562 & 764 & 2,233 & 7.4 & 5.7 & 1.7 & 0 \\
\hline 133 & Bistra & 3,320 & 3,078 & 243 & 6,632 & 22.0 & 20.4 & 1.6 & 3 \\
\hline 134 & Lovas & 3,306 & 3,356 & -50 & 1,214 & 4.0 & 4.1 & -0.1 & 3 \\
\hline 135 & Luka & 3,293 & 3,609 & -316 & 1,351 & 4.4 & 4.9 & -0.4 & 2 \\
\hline
\end{tabular}

NEWSLETTER 98 | K. OTT \& M. BRONIĆ | Budget outturns of Croatian municipalities, cities... | Institute of Public Finance 5 
Per capita (in HRK)

Total (in million HRK)

\begin{tabular}{|c|c|c|c|c|c|c|c|c|c|}
\hline & Municipality & $\begin{array}{c}\text { Reve- } \\
\text { nues }\end{array}$ & $\begin{array}{l}\text { Expen- } \\
\text { ditures }\end{array}$ & $\begin{array}{c}\text { Surplus } \\
\text { or } \\
\text { deficit }\end{array}$ & $\begin{array}{l}\text { Popula- } \\
\text { tion }\end{array}$ & $\begin{array}{c}\text { Reve- } \\
\text { nues }\end{array}$ & $\begin{array}{l}\text { Expen- } \\
\text { ditures }\end{array}$ & $\begin{array}{c}\text { Surplus } \\
\text { or } \\
\text { deficit }\end{array}$ & $\begin{array}{c}\text { Level of } \\
\text { budget } \\
\text { trans- } \\
\text { parency }\end{array}$ \\
\hline 136 & Udbina & 3,284 & 3,256 & 29 & 1,874 & 6.2 & 6.1 & 0.1 & 3 \\
\hline 137 & Gola & 3,281 & 4,995 & $-1,714$ & 2,431 & 8.0 & 12.1 & -4.2 & 0 \\
\hline 138 & Zagvozd & 3,260 & 3,627 & -367 & 1,188 & 3.9 & 4.3 & -0.4 & 0 \\
\hline 139 & Ervenik & 3,216 & 2,293 & 923 & 1,105 & 3.6 & 2.5 & 1.0 & 3 \\
\hline 140 & Peteranec & 3,196 & 2,788 & 408 & 2,704 & 8.6 & 7.5 & 1.1 & 0 \\
\hline 141 & Klakar & 3,192 & 2,006 & 1,186 & 2,319 & 7.4 & 4.7 & 2.8 & 4 \\
\hline 142 & Viljevo & 3,190 & 1,516 & 1,675 & 2,065 & 6.6 & 3.1 & 3.5 & 3 \\
\hline 143 & Gradac & 3,188 & 3,153 & 34 & 3,261 & 10.4 & 10.3 & 0.1 & 3 \\
\hline 144 & Majur & 3,163 & 2,447 & 716 & 1,185 & 3.7 & 2.9 & 0.8 & 0 \\
\hline 145 & Sirač & 3,099 & 2,986 & 112 & 2,218 & 6.9 & 6.6 & 0.2 & 1 \\
\hline 146 & Brod Moravice & 3,090 & 3,087 & 2 & 866 & 2.7 & 2.7 & 0.0 & 3 \\
\hline 147 & Smokvica & 3,079 & 2,709 & 370 & 916 & 2.8 & 2.5 & 0.3 & 1 \\
\hline 148 & Nijemci & 3,073 & 3,635 & -562 & 4,705 & 14.5 & 17.1 & -2.6 & 3 \\
\hline 149 & Jelenje & 3,071 & 2,952 & 118 & 5,344 & 16.4 & 15.8 & 0.6 & 3 \\
\hline 150 & Bibinje & 3,070 & 3,094 & -25 & 3,985 & 12.2 & 12.3 & -0.1 & 0 \\
\hline 151 & Lišane Ostrovičke & 3,063 & 3,721 & -658 & 698 & 2.1 & 2.6 & -0.5 & 0 \\
\hline 152 & Podstrana & 3,048 & 3,361 & -313 & 9,129 & 27.8 & 30.7 & -2.9 & 0 \\
\hline 153 & Ližnjan & 3,024 & 4,708 & $-1,684$ & 3,965 & 12.0 & 18.7 & -6.7 & 1 \\
\hline 154 & Višnjan & 3,013 & 3,433 & -420 & 2,274 & 6.9 & 7.8 & -1.0 & 2 \\
\hline 155 & Virje & 3,012 & 2,907 & 105 & 4,587 & 13.8 & 13.3 & 0.5 & 1 \\
\hline 156 & Antunovac & 3,001 & 2,467 & 534 & 3,703 & 11.1 & 9.1 & 2.0 & 3 \\
\hline 157 & Selca & 2,943 & 3,231 & -288 & 1,804 & 5.3 & 5.8 & -0.5 & 3 \\
\hline 158 & Gunja & 2,940 & 2,205 & 735 & 3,732 & 11.0 & 8.2 & 2.7 & 0 \\
\hline 159 & Posedarje & 2,908 & 2,812 & 96 & 3,607 & 10.5 & 10.1 & 0.3 & 0 \\
\hline 160 & Bilje & 2,906 & 3,635 & -729 & 5,642 & 16.4 & 20.5 & -4.1 & 1 \\
\hline 161 & Bosiljevo & 2,904 & 2,330 & 575 & 1,284 & 3.7 & 3.0 & 0.7 & 3 \\
\hline 162 & Čađavica & 2,876 & 2,614 & 263 & 2,009 & 5.8 & 5.3 & 0.5 & 0 \\
\hline 163 & Pušća & 2,866 & 2,517 & 349 & 2,700 & 7.7 & 6.8 & 0.9 & 0 \\
\hline 164 & Velika & 2,857 & 2,474 & 383 & 5,607 & 16.0 & 13.9 & 2.1 & 3 \\
\hline 165 & Cestica & 2,856 & 2,273 & 583 & 5,806 & 16.6 & 13.2 & 3.4 & 1 \\
\hline 166 & Dicmo & 2,851 & 3,314 & -463 & 2,802 & 8.0 & 9.3 & -1.3 & 0 \\
\hline 167 & Sukošan & 2,828 & 2,663 & 165 & 4,583 & 13.0 & 12.2 & 0.8 & 3 \\
\hline 168 & Lipovljani & 2,820 & 2,883 & -63 & 3,455 & 9.7 & 10.0 & -0.2 & 3 \\
\hline 169 & Lumbarda & 2,820 & 2,382 & 438 & 1,213 & 3.4 & 2.9 & 0.5 & 1 \\
\hline 170 & Lokvičići & 2,817 & 2,772 & 44 & 807 & 2.3 & 2.2 & 0.0 & 0 \\
\hline 171 & Seget & 2,812 & 2,293 & 518 & 4,854 & 13.6 & 11.1 & 2.5 & 2 \\
\hline 172 & Zagorska Sela & 2,798 & 3,110 & -312 & 996 & 2.8 & 3.1 & -0.3 & 0 \\
\hline 173 & Vela Luka & 2,768 & 2,231 & 537 & 4,137 & 11.4 & 9.2 & 2.2 & 3 \\
\hline 174 & Drnje & 2,759 & 2,702 & 57 & 1,863 & 5.1 & 5.0 & 0.1 & 2 \\
\hline 175 & Vrbanja & 2,753 & 2,926 & -172 & 3,940 & 10.8 & 11.5 & -0.7 & 3 \\
\hline 176 & Ražanac & 2,720 & 2,692 & 27 & 2,940 & 8.0 & 7.9 & 0.1 & 0 \\
\hline 177 & Križ & 2,712 & 2,998 & -285 & 6,963 & 18.9 & 20.9 & -2.0 & 3 \\
\hline 178 & Vrhovine & 2,683 & 2,982 & -299 & 1,381 & 3.7 & 4.1 & -0.4 & 3 \\
\hline 179 & Brinje & 2,675 & 2,917 & -242 & 3,256 & 8.7 & 9.5 & -0.8 & 1 \\
\hline 180 & Jagodnjak & 2,653 & 3,816 & $-1,163$ & 2,023 & 5.4 & 7.7 & -2.4 & 0 \\
\hline 181 & Bilice & 2,643 & 2,344 & 299 & 2,307 & 6.1 & 5.4 & 0.7 & 2 \\
\hline 182 & Gračac & 2,631 & 2,660 & -29 & 4,690 & 12.3 & 12.5 & -0.1 & 3 \\
\hline 183 & Dugi Rat & 2,609 & 2,123 & 486 & 7,092 & 18.5 & 15.1 & 3.4 & 0 \\
\hline 184 & Žumberak & 2,584 & 2,190 & 394 & 883 & 2.3 & 1.9 & 0.3 & 0 \\
\hline 185 & Dekanovec & 2,578 & 1,981 & 597 & 774 & 2.0 & 1.5 & 0.5 & 0 \\
\hline 186 & Brdovec & 2,567 & 2,583 & -16 & 11,134 & 28.6 & 28.8 & -0.2 & 0 \\
\hline 187 & Petlovac & 2,547 & 3,159 & -612 & 2,405 & 6.1 & 7.6 & -1.5 & 3 \\
\hline 188 & Promina & 2,535 & 2,299 & 236 & 1,136 & 2.9 & 2.6 & 0.3 & 1 \\
\hline 189 & Lasinja & 2,521 & 2,434 & 88 & 1,624 & 4.1 & 4.0 & 0.1 & 3 \\
\hline 190 & Stara Gradiška & 2,510 & 2,407 & 104 & 1,363 & 3.4 & 3.3 & 0.1 & 0 \\
\hline 191 & Hum na Sutli & 2,502 & 2,503 & -1 & 5,060 & 12.7 & 12.7 & 0.0 & 1 \\
\hline
\end{tabular}

6 NEWSLETTER 98 | K. OTT \& M. BRONIĆ | Budget outturns of Croatian municipalities, cities... | Institute of Public Finance 
Per capita (in HRK)

Total (in million HRK)

\begin{tabular}{|c|c|c|c|c|c|c|c|c|c|}
\hline & Municipality & $\begin{array}{c}\text { Reve- } \\
\text { nues }\end{array}$ & $\begin{array}{l}\text { Expen- } \\
\text { ditures }\end{array}$ & $\begin{array}{c}\text { Surplus } \\
\text { or } \\
\text { deficit }\end{array}$ & $\begin{array}{l}\text { Popula- } \\
\text { tion }\end{array}$ & $\begin{array}{c}\text { Reve- } \\
\text { nues }\end{array}$ & $\begin{array}{l}\text { Expen- } \\
\text { ditures }\end{array}$ & $\begin{array}{c}\text { Surplus } \\
\text { or } \\
\text { deficit }\end{array}$ & $\begin{array}{c}\text { Level of } \\
\text { budget } \\
\text { trans- } \\
\text { parency }\end{array}$ \\
\hline 192 & Đurmanec & 2,487 & 2,395 & 92 & 4,235 & 10.5 & 10.1 & 0.4 & 2 \\
\hline 193 & Gradište & 2,481 & 1,473 & 1,008 & 2,773 & 6.9 & 4.1 & 2.8 & 0 \\
\hline 194 & Tovarnik & 2,473 & 2,645 & -172 & 2,775 & 6.9 & 7.3 & -0.5 & 4 \\
\hline 195 & Semeljci & 2,472 & 2,499 & -28 & 4,362 & 10.8 & 10.9 & -0.1 & 2 \\
\hline 196 & Tounj & 2,469 & 1,659 & 810 & 1,150 & 2.8 & 1.9 & 0.9 & 4 \\
\hline 197 & Barban & 2,441 & 3,506 & $-1,065$ & 2,721 & 6.6 & 9.5 & -2.9 & 0 \\
\hline 198 & Josipdol & 2,425 & 2,316 & 109 & 3,773 & 9.2 & 8.7 & 0.4 & 2 \\
\hline 199 & Tordinci & 2,387 & 2,045 & 342 & 2,032 & 4.8 & 4.2 & 0.7 & 0 \\
\hline 200 & Gornji Kneginec & 2,369 & 1,670 & 699 & 5,349 & 12.7 & 8.9 & 3.7 & 1 \\
\hline 201 & Gornja Stubica & 2,367 & 2,531 & -164 & 5,284 & 12.5 & 13.4 & -0.9 & 0 \\
\hline 202 & Kalnik & 2,361 & 2,260 & 102 & 1,351 & 3.2 & 3.1 & 0.1 & 2 \\
\hline 203 & $\begin{array}{l}\text { Novigrad } \\
\text { Podravski }\end{array}$ & 2,360 & 2,304 & 56 & 2,872 & 6.8 & 6.6 & 0.2 & 2 \\
\hline 204 & Tompojevci & 2,357 & 2,669 & -313 & 1,565 & 3.7 & 4.2 & -0.5 & 2 \\
\hline 205 & Kravarsko & 2,341 & 2,115 & 227 & 1,987 & 4.7 & 4.2 & 0.5 & 2 \\
\hline 206 & Vladislavci & 2,337 & 2,368 & -32 & 1,882 & 4.4 & 4.5 & -0.1 & 2 \\
\hline 207 & Zdenci & 2,316 & 1,954 & 362 & 1,904 & 4.4 & 3.7 & 0.7 & 1 \\
\hline 208 & Cista Provo & 2,289 & 2,542 & -254 & 2,335 & 5.3 & 5.9 & -0.6 & 0 \\
\hline 209 & Otok (Split) & 2,282 & 2,077 & 205 & 5,474 & 12.5 & 11.4 & 1.1 & 0 \\
\hline 210 & Proložac & 2,277 & 2,888 & -611 & 3,802 & 8.7 & 11.0 & -2.3 & 0 \\
\hline 211 & Šandrovac & 2,275 & 2,390 & -115 & 1,776 & 4.0 & 4.2 & -0.2 & 3 \\
\hline 212 & Žminj & 2,271 & 2,515 & -244 & 3,483 & 7.9 & 8.8 & -0.8 & 3 \\
\hline 213 & $\begin{array}{l}\text { Podravska } \\
\text { Moslavina }\end{array}$ & 2,271 & 2,267 & 4 & 1,202 & 2.7 & 2.7 & 0.0 & 0 \\
\hline 214 & Unešić & 2,267 & 2,156 & 110 & 1,686 & 3.8 & 3.6 & 0.2 & 0 \\
\hline 215 & Rugvica & 2,264 & 1,870 & 394 & 7,871 & 17.8 & 14.7 & 3.1 & 0 \\
\hline 216 & Čeminac & 2,255 & 2,083 & 172 & 2,909 & 6.6 & 6.1 & 0.5 & 0 \\
\hline 217 & Sveti Petar u Šumi & 2,254 & 4,758 & $-2,504$ & 1,065 & 2.4 & 5.1 & -2.7 & 0 \\
\hline 218 & Veliki Grđevac & 2,251 & 2,108 & 144 & 2,849 & 6.4 & 6.0 & 0.4 & 0 \\
\hline 219 & Muć & 2,245 & 1,866 & 379 & 3,882 & 8.7 & 7.2 & 1.5 & 1 \\
\hline 220 & Konjščina & 2,242 & 2,620 & -378 & 3,790 & 8.5 & 9.9 & -1.4 & 3 \\
\hline 221 & Voćin & 2,235 & 3,020 & -785 & 2,382 & 5.3 & 7.2 & -1.9 & 0 \\
\hline 222 & Draž & 2,226 & 2,205 & 21 & 2,767 & 6.2 & 6.1 & 0.1 & 0 \\
\hline 223 & Zlatar Bistrica & 2,163 & 2,304 & -141 & 2,600 & 5.6 & 6.0 & -0.4 & 1 \\
\hline 224 & Ružić & 2,158 & 1,918 & 240 & 1,591 & 3.4 & 3.1 & 0.4 & 3 \\
\hline 225 & Šestanovac & 2,154 & 2,424 & -270 & 1,958 & 4.2 & 4.7 & -0.5 & 1 \\
\hline 226 & Lovreć & 2,147 & 2,146 & 1 & 1,699 & 3.6 & 3.6 & 0.0 & 1 \\
\hline 227 & Pokupsko & 2,138 & 2,259 & -121 & 2,224 & 4.8 & 5.0 & -0.3 & 0 \\
\hline 228 & Veliko Trojstvo & 2,137 & 1,949 & 188 & 2,741 & 5.9 & 5.3 & 0.5 & 0 \\
\hline 229 & Suhopolje & 2,130 & 1,989 & 142 & 6,683 & 14.2 & 13.3 & 0.9 & 1 \\
\hline 230 & Erdut & 2,123 & 2,075 & 48 & 7,308 & 15.5 & 15.2 & 0.4 & 2 \\
\hline 231 & $\begin{array}{l}\text { Sveti Juraj } \\
\text { na Bregu }\end{array}$ & 2,119 & 2,253 & -135 & 5,090 & 10.8 & 11.5 & -0.7 & 1 \\
\hline 232 & Dubravica & 2,119 & 2,723 & -604 & 1,437 & 3.0 & 3.9 & -0.9 & 2 \\
\hline 233 & Topusko & 2,118 & 2,334 & -216 & 2,985 & 6.3 & 7.0 & -0.6 & 3 \\
\hline 234 & Brckovljani & 2,105 & 1,932 & 174 & 6,837 & 14.4 & 13.2 & 1.2 & 0 \\
\hline 235 & $\begin{array}{l}\text { Privlaka } \\
\text { (Vinkovci) }\end{array}$ & 2,080 & 2,297 & -217 & 2,954 & 6.1 & 6.8 & -0.6 & 0 \\
\hline 236 & Kloštar Ivanić & 2,075 & 2,140 & -65 & 6,091 & 12.6 & 13.0 & -0.4 & 1 \\
\hline 237 & Novo Virje & 2,058 & 1,783 & 276 & 1,216 & 2.5 & 2.2 & 0.3 & 0 \\
\hline 238 & Orehovica & 2,043 & 1,795 & 247 & 2,685 & 5.5 & 4.8 & 0.7 & 0 \\
\hline 239 & Novi Golubovec & 2,042 & 1,758 & 284 & 996 & 2.0 & 1.8 & 0.3 & 0 \\
\hline 240 & Šenkovec & 2,040 & 2,106 & -66 & 2,879 & 5.9 & 6.1 & -0.2 & 0 \\
\hline 241 & Zmijavci & 2,039 & 2,158 & -120 & 2,048 & 4.2 & 4.4 & -0.2 & 1 \\
\hline 242 & Karojba & 2,028 & 2,819 & -791 & 1,438 & 2.9 & 4.1 & -1.1 & 0 \\
\hline 243 & Severin & 2,025 & 2,084 & -60 & 877 & 1.8 & 1.8 & -0.1 & 0 \\
\hline 244 & Generalski Stol & 2,019 & 1,846 & 172 & 2,642 & 5.3 & 4.9 & 0.5 & 3 \\
\hline
\end{tabular}

NEWSLETTER 98 | K. OTT \& M. BRONIĆ | Budget outturns of Croatian municipalities, cities... | Institute of Public Finance 7 
Per capita (in HRK)

Total (in million HRK)

\begin{tabular}{|c|c|c|c|c|c|c|c|c|c|}
\hline & Municipality & $\begin{array}{c}\text { Reve- } \\
\text { nues }\end{array}$ & $\begin{array}{l}\text { Expen- } \\
\text { ditures }\end{array}$ & $\begin{array}{c}\text { Surplus } \\
\text { or } \\
\text { deficit }\end{array}$ & $\begin{array}{l}\text { Popula- } \\
\text { tion }\end{array}$ & $\begin{array}{c}\text { Reve- } \\
\text { nues }\end{array}$ & $\begin{array}{l}\text { Expen- } \\
\text { ditures }\end{array}$ & $\begin{array}{c}\text { Surplus } \\
\text { or } \\
\text { deficit }\end{array}$ & $\begin{array}{c}\text { Level of } \\
\text { budget } \\
\text { trans- } \\
\text { parency }\end{array}$ \\
\hline 245 & Bednja & 2,012 & 1,943 & 68 & 3,992 & 8.0 & 7.8 & 0.3 & 0 \\
\hline 246 & Kraljevec na Sutli & 2,011 & 2,142 & -131 & 1,727 & 3.5 & 3.7 & -0.2 & 2 \\
\hline 247 & Marija Bistrica & 2,010 & 2,350 & -340 & 5,976 & 12.0 & 14.0 & -2.0 & 0 \\
\hline 248 & Oriovac & 2,007 & 1,671 & 336 & 5,824 & 11.7 & 9.7 & 2.0 & 1 \\
\hline 249 & Vuka & 2,001 & 1,643 & 358 & 1,200 & 2.4 & 2.0 & 0.4 & 3 \\
\hline 250 & Berek & 1,997 & 2,011 & -15 & 1,443 & 2.9 & 2.9 & 0.0 & 1 \\
\hline 251 & Šodolovci & 1,995 & 1,816 & 179 & 1,653 & 3.3 & 3.0 & 0.3 & 1 \\
\hline 252 & Gornja Rijeka & 1,986 & 1,711 & 275 & 1,779 & 3.5 & 3.0 & 0.5 & 2 \\
\hline 253 & Bedenica & 1,968 & 1,669 & 299 & 1,432 & 2.8 & 2.4 & 0.4 & 0 \\
\hline 254 & Davor & 1,959 & 2,343 & -384 & 3,015 & 5.9 & 7.1 & -1.2 & 0 \\
\hline 255 & Velika Ludina & 1,952 & 2,784 & -832 & 2,625 & 5.1 & 7.3 & -2.2 & 2 \\
\hline 256 & Popovac & 1,944 & 2,273 & -329 & 2,084 & 4.1 & 4.7 & -0.7 & 2 \\
\hline 257 & Škabrnja & 1,927 & 1,865 & 62 & 1,776 & 3.4 & 3.3 & 0.1 & 0 \\
\hline 258 & Krapinske Toplice & 1,919 & 1,971 & -52 & 5,367 & 10.3 & 10.6 & -0.3 & 3 \\
\hline 259 & Stari Jankovci & 1,913 & 1,884 & 28 & 4,405 & 8.4 & 8.3 & 0.1 & 2 \\
\hline 260 & Lekenik & 1,906 & 1,905 & 1 & 6,032 & 11.5 & 11.5 & 0.0 & 0 \\
\hline 261 & Tuhelj & 1,905 & 1,671 & 233 & 2,104 & 4.0 & 3.5 & 0.5 & 3 \\
\hline 262 & Kamanje & 1,887 & 2,409 & -522 & 891 & 1.7 & 2.1 & -0.5 & 3 \\
\hline 263 & Veliko Trgovišće & 1,881 & 1,998 & -117 & 4,945 & 9.3 & 9.9 & -0.6 & 1 \\
\hline 264 & Darda & 1,875 & 2,436 & -560 & 6,908 & 13.0 & 16.8 & -3.9 & 2 \\
\hline 265 & Lobor & 1,874 & 1,624 & 250 & 3,188 & 6.0 & 5.2 & 0.8 & 2 \\
\hline 266 & $\begin{array}{l}\text { Satnica } \\
\text { Đakovačka }\end{array}$ & 1,860 & 1,851 & 9 & 2,123 & 3.9 & 3.9 & 0.0 & 1 \\
\hline 267 & Zrinski Topolovac & 1,829 & 1,976 & -147 & 890 & 1.6 & 1.8 & -0.1 & 0 \\
\hline 268 & Klinča Sela & 1,820 & 1,568 & 252 & 5,231 & 9.5 & 8.2 & 1.3 & 0 \\
\hline 269 & Punitovci & 1,802 & 1,620 & 182 & 1,803 & 3.2 & 2.9 & 0.3 & 0 \\
\hline 270 & Podbablje & 1,802 & 1,444 & 357 & 4,680 & 8.4 & 6.8 & 1.7 & 1 \\
\hline 271 & Netretić & 1,796 & 1,552 & 244 & 2,862 & 5.1 & 4.4 & 0.7 & 1 \\
\hline 272 & Krnjak & 1,771 & 1,549 & 222 & 1,985 & 3.5 & 3.1 & 0.4 & 4 \\
\hline 273 & Slivno & 1,762 & 1,425 & 336 & 1,999 & 3.5 & 2.8 & 0.7 & 1 \\
\hline 274 & Cetingrad & 1,759 & 1,706 & 53 & 2,027 & 3.6 & 3.5 & 0.1 & 0 \\
\hline 275 & Fernandinovac & 1,759 & 1,901 & -143 & 1,750 & 3.1 & 3.3 & -0.2 & 1 \\
\hline 276 & $\begin{array}{l}\text { Koprivnički } \\
\text { Ivanec }\end{array}$ & 1,752 & 3,457 & $-1,705$ & 2,121 & 3.7 & 7.3 & -3.6 & 0 \\
\hline 277 & Hlebine & 1,751 & 1,519 & 232 & 1,304 & 2.3 & 2.0 & 0.3 & 2 \\
\hline 278 & Ernestinovo & 1,750 & 1,885 & -136 & 2,189 & 3.8 & 4.1 & -0.3 & 3 \\
\hline 279 & Kistanje & 1,748 & 1,905 & -157 & 3,481 & 6.1 & 6.6 & -0.5 & 2 \\
\hline 280 & Podgorač & 1,744 & 1,650 & 94 & 2,877 & 5.0 & 4.7 & 0.3 & 3 \\
\hline 281 & Kumrovec & 1,741 & 2,011 & -270 & 1,588 & 2.8 & 3.2 & -0.4 & 2 \\
\hline 282 & Jakovlje & 1,732 & 1,391 & 341 & 3,930 & 6.8 & 5.5 & 1.3 & 0 \\
\hline 283 & Čačinci & 1,720 & 1,838 & -118 & 2,802 & 4.8 & 5.2 & -0.3 & 0 \\
\hline 284 & Biskupija & 1,709 & 1,886 & -178 & 1,699 & 2.9 & 3.2 & -0.3 & 2 \\
\hline 285 & Hrvatska Dubica & 1,701 & 1,563 & 138 & 2,089 & 3.6 & 3.3 & 0.3 & 0 \\
\hline 286 & Sopje & 1,694 & 1,487 & 207 & 2,320 & 3.9 & 3.4 & 0.5 & 0 \\
\hline 287 & Sveti Ilija & 1,685 & 2,171 & -487 & 3,511 & 5.9 & 7.6 & -1.7 & 0 \\
\hline 288 & $\begin{array}{l}\text { Trnovec } \\
\text { Bartolovečki }\end{array}$ & 1,682 & 1,529 & 153 & 6,884 & 11.6 & 10.5 & 1.1 & 3 \\
\hline 289 & Gradina & 1,680 & 1,621 & 60 & 3,850 & 6.5 & 6.2 & 0.2 & 1 \\
\hline 290 & Budinščina & 1,674 & 1,651 & 23 & 2,503 & 4.2 & 4.1 & 0.1 & 0 \\
\hline 291 & Draganić & 1,673 & 1,730 & -57 & 2,741 & 4.6 & 4.7 & -0.2 & 1 \\
\hline 292 & Breznički Hum & 1,658 & 1,903 & -245 & 1,356 & 2.2 & 2.6 & -0.3 & 1 \\
\hline 293 & Lukač & 1,656 & 1,482 & 174 & 3,634 & 6.0 & 5.4 & 0.6 & 3 \\
\hline 294 & $\begin{array}{l}\text { Sveti Križ } \\
\text { Začretje }\end{array}$ & 1,654 & 1,535 & 119 & 6,165 & 10.2 & 9.5 & 0.7 & 4 \\
\hline 295 & Nuštar & 1,641 & 1,597 & 44 & 5,793 & 9.5 & 9.2 & 0.3 & 3 \\
\hline 296 & Pitomača & 1,639 & 1,567 & 72 & 10,059 & 16.5 & 15.8 & 0.7 & 2 \\
\hline 297 & Vojnić & 1,633 & 2,313 & -680 & 4,764 & 7.8 & 11.0 & -3.2 & 4 \\
\hline
\end{tabular}


Per capita (in HRK)

Total (in million HRK)

\begin{tabular}{|c|c|c|c|c|c|c|c|c|c|}
\hline & Municipality & $\begin{array}{c}\text { Reve- } \\
\text { nues }\end{array}$ & $\begin{array}{l}\text { Expen- } \\
\text { ditures }\end{array}$ & $\begin{array}{c}\text { Surplus } \\
\text { or } \\
\text { deficit }\end{array}$ & $\begin{array}{l}\text { Popula- } \\
\text { tion }\end{array}$ & $\begin{array}{c}\text { Reve- } \\
\text { nues }\end{array}$ & $\begin{array}{l}\text { Expen- } \\
\text { ditures }\end{array}$ & $\begin{array}{c}\text { Surplus } \\
\text { or } \\
\text { deficit }\end{array}$ & $\begin{array}{c}\text { Level of } \\
\text { budget } \\
\text { trans- } \\
\text { parency }\end{array}$ \\
\hline 298 & Bizovac & 1,622 & 1,491 & 131 & 4,507 & 7.3 & 6.7 & 0.6 & 4 \\
\hline 299 & Donji Lapac & 1,616 & 1,508 & 108 & 2,113 & 3.4 & 3.2 & 0.2 & 0 \\
\hline 300 & Čaglin & 1,615 & 1,331 & 284 & 2,723 & 4.4 & 3.6 & 0.8 & 0 \\
\hline 301 & Vođinci & 1,613 & 1,534 & 80 & 1,966 & 3.2 & 3.0 & 0.2 & 2 \\
\hline 302 & Jarmina & 1,611 & 1,430 & 181 & 2,458 & 4.0 & 3.5 & 0.4 & 3 \\
\hline 303 & Babina Greda & 1,611 & 1,822 & -211 & 3,572 & 5.8 & 6.5 & -0.8 & 1 \\
\hline 304 & Nedelišće & 1,611 & 1,620 & -10 & 11,975 & 19.3 & 19.4 & -0.1 & 1 \\
\hline 305 & Petrijevci & 1,594 & 1,593 & 2 & 2,870 & 4.6 & 4.6 & 0.0 & 3 \\
\hline 306 & Brestovac & 1,587 & 1,410 & 177 & 3,726 & 5.9 & 5.3 & 0.7 & 0 \\
\hline 307 & Goričan & 1,587 & 1,444 & 143 & 2,823 & 4.5 & 4.1 & 0.4 & 1 \\
\hline 308 & Bogdanovci & 1,581 & 1,424 & 157 & 1,960 & 3.1 & 2.8 & 0.3 & 3 \\
\hline 309 & Strahoninec & 1,577 & 1,100 & 477 & 2,682 & 4.2 & 2.9 & 1.3 & 1 \\
\hline 310 & Veliki Bukovec & 1,574 & 1,846 & -272 & 1,438 & 2.3 & 2.7 & -0.4 & 0 \\
\hline 311 & Dragalić & 1,568 & 1,456 & 112 & 1,361 & 2.1 & 2.0 & 0.2 & 3 \\
\hline 312 & Gvozd & 1,564 & 1,699 & -135 & 2,970 & 4.6 & 5.0 & -0.4 & 0 \\
\hline 313 & Podturen & 1,563 & 1,384 & 179 & 3,873 & 6.1 & 5.4 & 0.7 & 3 \\
\hline 314 & Velika Pisanica & 1,552 & 1,544 & 8 & 1,781 & 2.8 & 2.7 & 0.0 & 1 \\
\hline 315 & Ivanska & 1,550 & 1,814 & -264 & 2,911 & 4.5 & 5.3 & -0.8 & 1 \\
\hline 316 & Donja Dubrava & 1,526 & 1,572 & -45 & 1,920 & 2.9 & 3.0 & -0.1 & 0 \\
\hline 317 & Gundinci & 1,526 & 1,012 & 513 & 2,027 & 3.1 & 2.1 & 1.0 & 4 \\
\hline 318 & Oprisavci & 1,517 & 1,478 & 39 & 2,508 & 3.8 & 3.7 & 0.1 & 1 \\
\hline 319 & Donja Motičina & 1,515 & 1,432 & 84 & 1,652 & 2.5 & 2.4 & 0.1 & 3 \\
\hline 320 & Kula Norinska & 1,514 & 1,386 & 128 & 1,748 & 2.6 & 2.4 & 0.2 & 1 \\
\hline 321 & Plaški & 1,514 & 1,237 & 277 & 2,090 & 3.2 & 2.6 & 0.6 & 0 \\
\hline 322 & Gornji Bogićevci & 1,501 & 1,426 & 75 & 1,975 & 3.0 & 2.8 & 0.1 & 1 \\
\hline 323 & Velika Trnovitica & 1,500 & 1,358 & 143 & 1,370 & 2.1 & 1.9 & 0.2 & 0 \\
\hline 324 & Bedekovčina & 1,497 & 1,261 & 236 & 8,041 & 12.0 & 10.1 & 1.9 & 3 \\
\hline 325 & Garčin & 1,485 & 1,543 & -58 & 4,806 & 7.1 & 7.4 & -0.3 & 1 \\
\hline 326 & Žakanje & 1,471 & 1,287 & 185 & 1,889 & 2.8 & 2.4 & 0.3 & 1 \\
\hline 327 & Beretinec & 1,467 & 1,436 & 31 & 2,176 & 3.2 & 3.1 & 0.1 & 3 \\
\hline 328 & Đulovac & 1,463 & 1,275 & 188 & 3,245 & 4.7 & 4.1 & 0.6 & 1 \\
\hline 329 & Vinica & 1,461 & 1,396 & 65 & 3,389 & 5.0 & 4.7 & 0.2 & 0 \\
\hline 330 & Trnava & 1,447 & 2,410 & -963 & 1,600 & 2.3 & 3.9 & -1.5 & 0 \\
\hline 331 & $\begin{array}{l}\text { Sveti Martin } \\
\text { na Muri }\end{array}$ & 1,441 & 1,434 & 6 & 2,605 & 3.8 & 3.7 & 0.0 & 2 \\
\hline 332 & Hercegovac & 1,441 & 1,445 & -4 & 2,383 & 3.4 & 3.4 & 0.0 & 3 \\
\hline 333 & Cernik & 1,428 & 1,347 & 81 & 3,640 & 5.2 & 4.9 & 0.3 & 3 \\
\hline 334 & Dvor & 1,422 & 1,287 & 135 & 5,570 & 7.9 & 7.2 & 0.8 & 2 \\
\hline 335 & Gorjani & 1,421 & 1,176 & 246 & 1,591 & 2.3 & 1.9 & 0.4 & 0 \\
\hline 336 & Sunja & 1,414 & 1,248 & 166 & 5,748 & 8.1 & 7.2 & 1.0 & 1 \\
\hline 337 & Galovac & 1,412 & 1,199 & 213 & 1,234 & 1.7 & 1.5 & 0.3 & 0 \\
\hline 338 & Velika Kopanica & 1,410 & 1,482 & -72 & 3,308 & 4.7 & 4.9 & -0.2 & 1 \\
\hline 339 & Klenovnik & 1,409 & 1,783 & -374 & 2,022 & 2.8 & 3.6 & -0.8 & 0 \\
\hline 340 & Vrpolje & 1,400 & 1,371 & 29 & 3,521 & 4.9 & 4.8 & 0.1 & 0 \\
\hline 341 & Okučani & 1,396 & 1,432 & -35 & 3,447 & 4.8 & 4.9 & -0.1 & 1 \\
\hline 342 & Sveta Marija & 1,395 & 1,303 & 92 & 2,317 & 3.2 & 3.0 & 0.2 & 1 \\
\hline 343 & Petrijanec & 1,383 & 1,179 & 204 & 4,812 & 6.7 & 5.7 & 1.0 & 0 \\
\hline 344 & Donji Vidovec & 1,381 & 1,344 & 37 & 1,399 & 1.9 & 1.9 & 0.1 & 0 \\
\hline 345 & Podcrkavlje & 1,379 & 1,283 & 96 & 2,553 & 3.5 & 3.3 & 0.2 & 2 \\
\hline 346 & Bošnjaci & 1,372 & 1,351 & 21 & 3,901 & 5.4 & 5.3 & 0.1 & 0 \\
\hline 347 & Mali Bukovec & 1,367 & 1,258 & 109 & 2,212 & 3.0 & 2.8 & 0.2 & 3 \\
\hline 348 & Koška & 1,365 & 1,280 & 85 & 3,980 & 5.4 & 5.1 & 0.3 & 1 \\
\hline 349 & Viškovci & 1,361 & 1,265 & 95 & 1,906 & 2.6 & 2.4 & 0.2 & 0 \\
\hline 350 & Trpinja & 1,358 & 1,329 & 29 & 5,572 & 7.6 & 7.4 & 0.2 & 0 \\
\hline 351 & Rakovec & 1,353 & 1,158 & 195 & 1,252 & 1.7 & 1.5 & 0.2 & 3 \\
\hline 352 & Vrbje & 1,353 & 1,088 & 265 & 2,215 & 3.0 & 2.4 & 0.6 & 0 \\
\hline
\end{tabular}

NEWSLETTER 98 | K. OTT \& M. BRONIĆ | Budget outturns of Croatian municipalities, cities... | Institute of Public Finance 9 
Per capita (in HRK)

Total (in million HRK)

\begin{tabular}{|c|c|c|c|c|c|c|c|c|c|}
\hline & Municipality & $\begin{array}{c}\text { Reve- } \\
\text { nues }\end{array}$ & $\begin{array}{l}\text { Expen- } \\
\text { ditures }\end{array}$ & $\begin{array}{c}\text { Surplus } \\
\text { or } \\
\text { deficit }\end{array}$ & $\begin{array}{l}\text { Popula- } \\
\text { tion }\end{array}$ & $\begin{array}{c}\text { Reve- } \\
\text { nues }\end{array}$ & $\begin{array}{l}\text { Expen- } \\
\text { ditures }\end{array}$ & $\begin{array}{c}\text { Surplus } \\
\text { or } \\
\text { deficit }\end{array}$ & $\begin{array}{c}\text { Level of } \\
\text { budget } \\
\text { trans- } \\
\text { parency }\end{array}$ \\
\hline 353 & Feričanci & 1,352 & 1,299 & 53 & 2,134 & 2.9 & 2.8 & 0.1 & 3 \\
\hline 354 & Donji Kukuruzari & 1,347 & 1,404 & -57 & 1,634 & 2.2 & 2.3 & -0.1 & 3 \\
\hline 355 & Donji Kraljevec & 1,340 & 1,187 & 153 & 4,659 & 6.2 & 5.5 & 0.7 & 3 \\
\hline 356 & Pribislavec & 1,331 & 1,387 & -56 & 3,136 & 4.2 & 4.4 & -0.2 & 1 \\
\hline 357 & Mikleuš & 1,329 & 1,315 & 14 & 1,464 & 1.9 & 1.9 & 0.0 & 2 \\
\hline 358 & Sračinec & 1,327 & 1,234 & 93 & 4,842 & 6.4 & 6.0 & 0.4 & 2 \\
\hline 359 & Andrijaševci & 1,311 & 1,143 & 168 & 4,075 & 5.3 & 4.7 & 0.7 & 3 \\
\hline 360 & Nova Rača & 1,306 & 1,163 & 142 & 3,433 & 4.5 & 4.0 & 0.5 & 2 \\
\hline 361 & Kotoriba & 1,290 & 1,203 & 87 & 3,224 & 4.2 & 3.9 & 0.3 & 3 \\
\hline 362 & Domašinec & 1,287 & 982 & 305 & 2,251 & 2.9 & 2.2 & 0.7 & 1 \\
\hline 363 & Dežanovac & 1,287 & 1,795 & -508 & 2,715 & 3.5 & 4.9 & -1.4 & 0 \\
\hline 364 & Runovići & 1,286 & 1,421 & -135 & 2,416 & 3.1 & 3.4 & -0.3 & 1 \\
\hline 365 & Čepin & 1,286 & 1,166 & 120 & 11,599 & 14.9 & 13.5 & 1.4 & 0 \\
\hline 366 & Donji Andrijevci & 1,282 & 1,117 & 164 & 3,709 & 4.8 & 4.1 & 0.6 & 1 \\
\hline 367 & Kloštar Podravski & 1,280 & 1,375 & -96 & 3,306 & 4.2 & 4.5 & -0.3 & 0 \\
\hline 368 & Slavonski Šamac & 1,279 & 1,208 & 70 & 2,169 & 2.8 & 2.6 & 0.2 & 2 \\
\hline 369 & Brodski Stupnik & 1,278 & 1,576 & -298 & 3,036 & 3.9 & 4.8 & -0.9 & 1 \\
\hline 370 & Krašić & 1,274 & 1,279 & -5 & 2,640 & 3.4 & 3.4 & 0.0 & 3 \\
\hline 371 & Stari Mikanovci & 1,260 & 1,251 & 9 & 2,956 & 3.7 & 3.7 & 0.0 & 2 \\
\hline 372 & Sveti Đurđ & 1,257 & 1,367 & -110 & 3,804 & 4.8 & 5.2 & -0.4 & 1 \\
\hline 373 & Belica & 1,245 & 1,167 & 78 & 3,176 & 4.0 & 3.7 & 0.2 & 4 \\
\hline 374 & Špišić Bukovica & 1,243 & 1,228 & 15 & 4,221 & 5.2 & 5.2 & 0.1 & 0 \\
\hline 375 & Kaptol & 1,240 & 1,283 & -43 & 3,472 & 4.3 & 4.5 & -0.1 & 0 \\
\hline 376 & Đelekovec & 1,240 & 1,200 & 41 & 1,533 & 1.9 & 1.8 & 0.1 & 2 \\
\hline 377 & Gradec & 1,208 & 1,206 & 2 & 3,681 & 4.4 & 4.4 & 0.0 & 0 \\
\hline 378 & Mihovljan & 1,204 & 1,077 & 127 & 1,938 & 2.3 & 2.1 & 0.2 & 1 \\
\hline 379 & Marijanci & 1,196 & 1,368 & -172 & 2,405 & 2.9 & 3.3 & -0.4 & 1 \\
\hline 380 & Pojezerje & 1,187 & 2,243 & $-1,056$ & 991 & 1.2 & 2.2 & -1.0 & 0 \\
\hline 381 & Bukovlje & 1,185 & 1,009 & 176 & 3,108 & 3.7 & 3.1 & 0.5 & 0 \\
\hline 382 & Štrigova & 1,174 & 1,033 & 141 & 2,766 & 3.2 & 2.9 & 0.4 & 1 \\
\hline 383 & Gornja Vrba & 1,170 & 1,032 & 138 & 2,512 & 2.9 & 2.6 & 0.3 & 1 \\
\hline 384 & Vidovec & 1,167 & 1,257 & -90 & 5,425 & 6.3 & 6.8 & -0.5 & 2 \\
\hline 385 & Gornji Mihaljevec & 1,160 & 915 & 245 & 1,917 & 2.2 & 1.8 & 0.5 & 1 \\
\hline 386 & Hrašćina & 1,142 & 982 & 160 & 1,617 & 1.8 & 1.6 & 0.3 & 0 \\
\hline 387 & Orle & 1,141 & 1,155 & -14 & 1,975 & 2.3 & 2.3 & 0.0 & 1 \\
\hline 388 & Staro Petrovo Selo & 1,132 & 1,035 & 97 & 5,186 & 5.9 & 5.4 & 0.5 & 2 \\
\hline 389 & Ivankovo & 1,126 & 1,102 & 24 & 8,006 & 9.0 & 8.8 & 0.2 & 3 \\
\hline 390 & Martinska Ves & 1,125 & 1,007 & 118 & 3,488 & 3.9 & 3.5 & 0.4 & 3 \\
\hline 391 & Kapela & 1,118 & 1,733 & -615 & 2,984 & 3.3 & 5.2 & -1.8 & 3 \\
\hline 392 & Sikirevci & 1,111 & 871 & 240 & 2,476 & 2.8 & 2.2 & 0.6 & 0 \\
\hline 393 & Breznica & 1,101 & 1,228 & -127 & 2,200 & 2.4 & 2.7 & -0.3 & 0 \\
\hline 394 & Levanjska Varoš & 1,084 & 1,491 & -407 & 1,194 & 1.3 & 1.8 & -0.5 & 0 \\
\hline 395 & Cerna & 1,083 & 901 & 182 & 4,595 & 5.0 & 4.1 & 0.8 & 0 \\
\hline 396 & Đurđenovac & 1,079 & 973 & 107 & 6,750 & 7.3 & 6.6 & 0.7 & 0 \\
\hline 397 & Selnica & 1,050 & 1,073 & -22 & 2,991 & 3.1 & 3.2 & -0.1 & 1 \\
\hline 398 & Mače & 1,044 & 880 & 164 & 2,534 & 2.6 & 2.2 & 0.4 & 3 \\
\hline 399 & Jakšić & 1,043 & 1,875 & -833 & 4,058 & 4.2 & 7.6 & -3.4 & 3 \\
\hline 400 & Martijanec & 1,039 & 1,169 & -130 & 3,843 & 4.0 & 4.5 & -0.5 & 1 \\
\hline 401 & Končanica & 1,028 & 922 & 106 & 2,360 & 2.4 & 2.2 & 0.3 & 0 \\
\hline 402 & Markušica & 1,028 & 921 & 107 & 2,555 & 2.6 & 2.4 & 0.3 & 3 \\
\hline 403 & Nova Kapela & 1,028 & 955 & 73 & 4,227 & 4.3 & 4.0 & 0.3 & 0 \\
\hline 404 & Vratišinec & 1,025 & 850 & 174 & 1,984 & 2.0 & 1.7 & 0.3 & 0 \\
\hline 405 & Desinić & 1,019 & 966 & 54 & 2,933 & 3.0 & 2.8 & 0.2 & 1 \\
\hline 406 & Petrovsko & 1,018 & 1,072 & -53 & 2,656 & 2.7 & 2.8 & -0.1 & 0 \\
\hline 407 & Štefanje & 994 & 839 & 155 & 2,030 & 2.0 & 1.7 & 0.3 & 1 \\
\hline 408 & Maruševec & 988 & 1,162 & -174 & 6,381 & 6.3 & 7.4 & -1.1 & 3 \\
\hline
\end{tabular}

IO NEWSLETTER 98 | K. OTT \& M. BRONIĆ | Budget outturns of Croatian municipalities, cities... | Institute of Public Finance 
Per capita (in HRK)

Total (in million HRK)

\begin{tabular}{|c|c|c|c|c|c|c|c|c|c|}
\hline & Municipality & $\begin{array}{c}\text { Reve- } \\
\text { nues }\end{array}$ & $\begin{array}{l}\text { Expen- } \\
\text { ditures }\end{array}$ & $\begin{array}{c}\text { Surplus } \\
\text { or } \\
\text { deficit }\end{array}$ & $\begin{array}{l}\text { Popula- } \\
\text { tion }\end{array}$ & $\begin{array}{l}\text { Reve- } \\
\text { nues }\end{array}$ & $\begin{array}{l}\text { Expen- } \\
\text { ditures }\end{array}$ & $\begin{array}{c}\text { Surplus } \\
\text { or } \\
\text { deficit }\end{array}$ & $\begin{array}{l}\text { Level of } \\
\text { budget } \\
\text { trans- } \\
\text { parency }\end{array}$ \\
\hline 409 & Mala Subotica & 988 & 875 & 113 & 5,452 & 5.4 & 4.8 & 0.6 & 2 \\
\hline 410 & Borovo & 984 & 914 & 70 & 5,056 & 5.0 & 4.6 & 0.4 & 1 \\
\hline 411 & Donja Voća & 970 & 724 & 246 & 2,443 & 2.4 & 1.8 & 0.6 & 0 \\
\hline 412 & Rasinja & 969 & 929 & 40 & 3,267 & 3.2 & 3.0 & 0.1 & 3 \\
\hline 413 & Sokolovac & 938 & 974 & -37 & 3,417 & 3.2 & 3.3 & -0.1 & 2 \\
\hline 414 & Zažablje & 922 & 849 & 73 & 757 & 0.7 & 0.6 & 0.1 & 0 \\
\hline 415 & Farkaševac & 917 & 942 & -25 & 1,937 & 1.8 & 1.8 & 0.0 & 1 \\
\hline 416 & Bebrina & 900 & 860 & 40 & 3,252 & 2.9 & 2.8 & 0.1 & 1 \\
\hline 417 & Rovišće & 899 & 824 & 74 & 4,822 & 4.3 & 4.0 & 0.4 & 0 \\
\hline 418 & Dubrava & 898 & 1,065 & -167 & 5,245 & 4.7 & 5.6 & -0.9 & 1 \\
\hline 419 & Visoko & 891 & 859 & 32 & 1,518 & 1.4 & 1.3 & 0.0 & 0 \\
\hline 420 & Sibinj & 875 & 750 & 125 & 6,895 & 6.0 & 5.2 & 0.9 & 1 \\
\hline 421 & Sveti Ivan Žabno & 863 & 750 & 114 & 5,222 & 4.5 & 3.9 & 0.6 & 1 \\
\hline 422 & Negoslavci & 828 & 871 & -43 & 1,463 & 1.2 & 1.3 & -0.1 & 1 \\
\hline 423 & Strizivojna & 802 & 752 & 50 & 2,525 & 2.0 & 1.9 & 0.1 & 0 \\
\hline 424 & Rešetari & 800 & 777 & 23 & 4,753 & 3.8 & 3.7 & 0.1 & 2 \\
\hline 425 & Drenje & 722 & 752 & -29 & 2,700 & 2.0 & 2.0 & -0.1 & 0 \\
\hline 426 & Preseka & 716 & 766 & -50 & 1,448 & 1.0 & 1.1 & -0.1 & 0 \\
\hline 427 & Štitar & 686 & 620 & 65 & 2,129 & 1.5 & 1.3 & 0.1 & 1 \\
\hline 428 & $\begin{array}{l}\text { Sveti Petar } \\
\text { Orehovec }\end{array}$ & 550 & 967 & -417 & 4,583 & 2.5 & 4.4 & -1.9 & 3 \\
\hline
\end{tabular}

Source: The authors, based on the Ministry of Finance (2015) and CBS (2015); and Ott, Bronić and Petrušić (2015).

Table 2

Budget outturns of cities, $2 \mathrm{OI} 4$

Per capita ( in HRK)

Gity

\section{Reve- \\ nues}

\section{Expen-
ditures}

\section{Surplus}

deficit

Popula-
tion

Total (in million HRK)

\begin{tabular}{|c|c|c|c|c|c|c|c|c|c|}
\hline 1 & Novalja & 11,659 & 11,252 & 407 & 3,663 & 43 & 41 & 1 & 3 \\
\hline 2 & Vis & 10,534 & 9,741 & 793 & 1,934 & 20 & 19 & 2 & 2 \\
\hline 3 & Vrlika & 10,466 & 10,203 & 264 & 2,177 & 23 & 22 & 1 & 1 \\
\hline 4 & Krk & 10,450 & 9,403 & 1,047 & 6,281 & 66 & 59 & 7 & 4 \\
\hline 5 & Rovinj & 9,976 & 8,016 & 1,960 & 14,294 & 143 & 115 & 28 & 1 \\
\hline 6 & Opatija & 9,920 & 10,295 & -375 & 11,659 & 116 & 120 & -4 & 5 \\
\hline 7 & Poreč & 9,395 & 6,923 & 2,471 & 16,696 & 157 & 116 & 41 & 4 \\
\hline 8 & Supetar & 8,908 & 6,477 & 2,430 & 4,074 & 36 & 26 & 10 & 1 \\
\hline 9 & Novigrad (Istra) & 8,900 & 8,519 & 381 & 4,345 & 39 & 37 & 2 & 4 \\
\hline 10 & Dubrovnik & 8,807 & 9,137 & -330 & 42,615 & 375 & 389 & -14 & 5 \\
\hline 11 & Zagreb & 8,418 & 9,662 & $-1,244$ & 790,017 & 6,651 & 7,633 & -983 & 5 \\
\hline 12 & Mali Lošinj & 8,208 & 8,256 & -48 & 8,116 & 67 & 67 & 0 & 3 \\
\hline 13 & Umag & 8,088 & 7,681 & 408 & 13,467 & 109 & 103 & 5 & 4 \\
\hline 14 & Hvar & 7,987 & 8,689 & -702 & 4,251 & 34 & 37 & -3 & 3 \\
\hline 15 & Cres & 7,740 & 7,940 & -200 & 2,879 & 22 & 23 & -1 & 4 \\
\hline 16 & Biograd na Moru & 7,302 & 7,173 & 129 & 5,569 & 41 & 40 & 1 & 2 \\
\hline 17 & Nin & 7,049 & 6,788 & 261 & 2,744 & 19 & 19 & 1 & 0 \\
\hline 18 & Lipik & 7,009 & 6,171 & 839 & 6,170 & 43 & 38 & 5 & 4 \\
\hline 19 & Pazin & 6,944 & 8,504 & $-1,560$ & 8,638 & 60 & 73 & -13 & 5 \\
\hline 20 & Crikvenica & 6,914 & 5,143 & 1,771 & 11,122 & 77 & 57 & 20 & 5 \\
\hline 21 & Buzet & 6,321 & 5,858 & 463 & 6,133 & 39 & 36 & 3 & 5 \\
\hline 22 & Stari Grad & 6,242 & 6,781 & -539 & 2,781 & 17 & 19 & -1 & 0 \\
\hline 23 & Vodnjan & 6,238 & 6,529 & -292 & 6,119 & 38 & 40 & -2 & 1 \\
\hline
\end{tabular}

\section{Surplus Level of}

or budget

deficit trans-

parency 
Per capita ( in HRK)

\begin{tabular}{|c|c|c|c|c|c|c|c|c|c|}
\hline & City & $\begin{array}{c}\text { Reve- } \\
\text { nues }\end{array}$ & $\begin{array}{l}\text { Expen- } \\
\text { ditures }\end{array}$ & $\begin{array}{c}\text { Surplus } \\
\text { or } \\
\text { deficit }\end{array}$ & $\begin{array}{l}\text { Popula- } \\
\text { tion }\end{array}$ & $\begin{array}{c}\text { Reve- } \\
\text { nues }\end{array}$ & $\begin{array}{l}\text { Expen- } \\
\text { ditures }\end{array}$ & $\begin{array}{c}\text { Surplus } \\
\text { or } \\
\text { deficit }\end{array}$ & $\begin{array}{c}\text { Level of } \\
\text { budget } \\
\text { trans- } \\
\text { parency }\end{array}$ \\
\hline 24 & Korčula & 6,143 & 5,999 & 144 & 5,663 & 35 & 34 & 1 & 3 \\
\hline 25 & Pakrac & 5,894 & 5,348 & 546 & 8,460 & 50 & 45 & 5 & 3 \\
\hline 26 & Bakar & 5,826 & 5,421 & 404 & 8,279 & 48 & 45 & 3 & 3 \\
\hline 27 & Labin & 5,790 & 5,534 & 257 & 11,642 & 67 & 64 & 3 & 4 \\
\hline 28 & Novi Vinodolski & 5,583 & 5,248 & 335 & 5,113 & 29 & 27 & 2 & 3 \\
\hline 29 & Senj & 5,469 & 3,639 & 1,830 & 7,182 & 39 & 26 & 13 & 3 \\
\hline 30 & Vodice & 5,459 & 4,467 & 991 & 8,875 & 48 & 40 & 9 & 5 \\
\hline 31 & Gospić & 5,322 & 5,252 & 70 & 12,745 & 68 & 67 & 1 & 0 \\
\hline 32 & Pag & 5,282 & 4,594 & 688 & 3,846 & 20 & 18 & 3 & 0 \\
\hline 33 & Rijeka & 5,230 & 4,919 & 312 & 128,624 & 673 & 633 & 40 & 5 \\
\hline 34 & Sveta Nedjelja & 5,093 & 3,752 & 1,341 & 18,059 & 92 & 68 & 24 & 4 \\
\hline 35 & Zaprešić & 4,924 & 4,412 & 513 & 25,223 & 124 & 111 & 13 & 4 \\
\hline 36 & Delnice & 4,906 & 5,253 & -346 & 5,952 & 29 & 31 & -2 & 3 \\
\hline 37 & Pula & 4,875 & 4,799 & 76 & 57,460 & 280 & 276 & 4 & 5 \\
\hline 38 & Makarska & 4,863 & 4,397 & 466 & 13,834 & 67 & 61 & 6 & 3 \\
\hline 39 & Vukovar & 4,763 & 3,447 & 1,316 & 27,683 & 132 & 95 & 36 & 4 \\
\hline 40 & Varaždin & 4,685 & 4,645 & 40 & 46,946 & 220 & 218 & 2 & 4 \\
\hline 41 & Zadar & 4,676 & 4,534 & 142 & 75,062 & 351 & 340 & 11 & 4 \\
\hline 42 & Komiža & 4,606 & 4,090 & 516 & 1,526 & 7 & 6 & 1 & 3 \\
\hline 43 & Samobor & 4,513 & 3,979 & 533 & 37,633 & 170 & 150 & 20 & 2 \\
\hline 44 & Rab & 4,370 & 5,231 & -861 & 8,065 & 35 & 42 & -7 & 1 \\
\hline 45 & Trogir & 4,174 & 3,649 & 525 & 13,192 & 55 & 48 & 7 & 3 \\
\hline 46 & Buje & 4,135 & 4,400 & -265 & 5,182 & 21 & 23 & -1 & 4 \\
\hline 47 & Split & 4,095 & 3,990 & 105 & 178,102 & 729 & 711 & 19 & 5 \\
\hline 48 & Šibenik & 3,967 & 4,278 & -310 & $\begin{array}{r}46,332 \\
\end{array}$ & 184 & 198 & -14 & 5 \\
\hline 49 & Orahovica & 3,950 & 2,885 & 1,065 & 5,304 & 21 & 15 & 6 & 3 \\
\hline 50 & Zabok & 3,929 & 3,827 & 103 & 8,994 & 35 & 34 & 1 & 2 \\
\hline 51 & Obrovac & 3,913 & 4,096 & -184 & 4,323 & 17 & 18 & -1 & 1 \\
\hline 52 & Virovitica & 3,895 & 3,295 & 600 & 21,291 & 83 & 70 & 13 & 3 \\
\hline 53 & Koprivnica & 3,817 & 4,453 & -636 & 30,854 & 118 & 137 & -20 & 5 \\
\hline 54 & Karlovac & 3,782 & 4,059 & -277 & 55,705 & 211 & 226 & -15 & 4 \\
\hline 55 & Čakovec & 3,762 & 3,636 & 126 & 27,104 & 102 & 99 & 3 & 5 \\
\hline 56 & Slunj & 3,694 & 3,557 & 138 & 5,076 & 19 & 18 & 1 & 4 \\
\hline 57 & Belišće & 3,627 & 5,029 & $-1,401$ & 10,825 & 39 & 54 & -15 & 0 \\
\hline 58 & Velika Gorica & 3,619 & 3,901 & -282 & 63,517 & 230 & 248 & -18 & 4 \\
\hline 59 & Čabar & 3,595 & 4,139 & -544 & 3,770 & 14 & 16 & -2 & 3 \\
\hline 60 & Vrbovsko & 3,592 & 3,288 & 305 & 5,076 & 18 & 17 & 2 & 1 \\
\hline 61 & Osijek & 3,540 & 3,546 & -6 & 108,048 & 382 & 383 & -1 & 5 \\
\hline 62 & Sisak & 3,517 & 3,471 & 46 & 47,768 & 168 & 166 & 2 & 0 \\
\hline 63 & Ivanić-Grad & 3,454 & 3,087 & 366 & 14,548 & 50 & 45 & 5 & 4 \\
\hline 64 & Kutina & 3,450 & 3,121 & 329 & 22,760 & 79 & 71 & 7 & 2 \\
\hline 65 & Solin & 3,447 & 3,657 & -210 & 23,926 & 82 & 88 & -5 & 3 \\
\hline 66 & Beli Manastir & 3,430 & 3,290 & 140 & 10,068 & 35 & 33 & 1 & 0 \\
\hline 67 & Omiš & 3,411 & 3,746 & -336 & 14,936 & 51 & 56 & -5 & 4 \\
\hline 68 & Đurđevac & 3,312 & 2,948 & 364 & 8,264 & 27 & 24 & 3 & 0 \\
\hline 69 & Kraljevica & 3,231 & 3,235 & -4 & 4,618 & 15 & 15 & 0 & 4 \\
\hline 70 & Kastav & 3,006 & 2,742 & 264 & 10,440 & 31 & 29 & 3 & 3 \\
\hline 71 & Drniš & 2,984 & 3,623 & -639 & 7,498 & 22 & 27 & -5 & 4 \\
\hline 72 & Križevci & 2,934 & 2,705 & 229 & 21,122 & 62 & 57 & 5 & 2 \\
\hline 73 & Donja Stubica & 2,844 & 2,853 & -9 & 5,680 & 16 & 16 & 0 & 3 \\
\hline 74 & Jastrebarsko & 2,839 & 3,610 & -771 & 15,866 & 45 & 57 & -12 & 4 \\
\hline 75 & Ogulin & 2,833 & 2,715 & 118 & 13,915 & 39 & 38 & 2 & 4 \\
\hline 76 & Skradin & 2,828 & 3,884 & $-1,056$ & 3,825 & 11 & 15 & -4 & 1 \\
\hline 77 & Petrinja & 2,799 & 2,912 & -113 & 24,671 & 69 & 72 & -3 & 0 \\
\hline
\end{tabular}


Per capita ( in HRK)

\begin{tabular}{|c|c|c|c|c|c|c|c|c|c|}
\hline & City & $\begin{array}{c}\text { Reve- } \\
\text { nues }\end{array}$ & $\begin{array}{l}\text { Expen- } \\
\text { ditures }\end{array}$ & $\begin{array}{c}\text { Surplus } \\
\text { or } \\
\text { deficit }\end{array}$ & $\begin{array}{l}\text { Popula- } \\
\text { tion }\end{array}$ & $\begin{array}{c}\text { Reve- } \\
\text { nues }\end{array}$ & $\begin{array}{l}\text { Expen- } \\
\text { ditures }\end{array}$ & $\begin{array}{c}\text { Surplus } \\
\text { or } \\
\text { deficit }\end{array}$ & $\begin{array}{c}\text { Level of } \\
\text { budget } \\
\text { trans- } \\
\text { parency }\end{array}$ \\
\hline 78 & Otočac & 2,767 & 2,071 & 697 & 9,778 & 27 & 20 & 7 & 0 \\
\hline 79 & Kaštela & 2,764 & 2,637 & 127 & 38,667 & 107 & 102 & 5 & 4 \\
\hline 80 & Vinkovci & 2,756 & 2,766 & -11 & 35,312 & 97 & 98 & 0 & 0 \\
\hline 81 & Požega & 2,749 & 2,603 & 146 & 26,248 & 72 & 68 & 4 & 1 \\
\hline 82 & Bjelovar & 2,691 & 2,706 & -15 & 40,276 & 108 & 109 & -1 & 4 \\
\hline 83 & Glina & 2,683 & 2,209 & 474 & 9,283 & 25 & 21 & 4 & 4 \\
\hline 84 & Novska & 2,658 & 2,598 & 60 & 13,518 & 36 & 35 & 1 & 4 \\
\hline 85 & Županja & 2,647 & 2,479 & 168 & 12,090 & 32 & 30 & 2 & 4 \\
\hline 86 & Ilok & 2,625 & 2,083 & 542 & 6,767 & 18 & 14 & 4 & 2 \\
\hline 87 & Ludbreg & 2,590 & 2,517 & 73 & 8,478 & 22 & 21 & 1 & 4 \\
\hline 88 & Imotski & 2,588 & 2,717 & -129 & 10,764 & 28 & 29 & -1 & 1 \\
\hline 89 & Popovača & 2,553 & 3,028 & -475 & 11,905 & 30 & 36 & -6 & 2 \\
\hline 90 & $\begin{array}{l}\text { Hrvatska } \\
\text { Kostajnica }\end{array}$ & 2,549 & 1,930 & 619 & 2,756 & 7 & 5 & 2 & 3 \\
\hline 91 & Prelog & 2,527 & 2,890 & -363 & 7,815 & 20 & 23 & -3 & 4 \\
\hline 92 & Lepoglava & 2,520 & 2,475 & 45 & 8,283 & 21 & 21 & 0 & 4 \\
\hline 93 & Našice & 2,515 & 2,182 & 332 & 16,224 & 41 & 35 & 5 & 4 \\
\hline 94 & Klanjec & 2,495 & 2,081 & 414 & 2,915 & 7 & 6 & 1 & 1 \\
\hline 95 & Vrgorac & 2,482 & 2,869 & -387 & 6,572 & 16 & 19 & -3 & 0 \\
\hline 96 & Grubišno Polje & 2,465 & 3,284 & -819 & 6,478 & 16 & 21 & -5 & 1 \\
\hline 97 & Opuzen & 2,461 & 2,937 & -477 & 3,254 & 8 & 10 & -2 & 2 \\
\hline 98 & Benkovac & 2,449 & 2,165 & 284 & 11,026 & 27 & 24 & 3 & 3 \\
\hline 99 & Krapina & 2,420 & 2,304 & 116 & 12,480 & 30 & 29 & 1 & 4 \\
\hline 100 & Knin & 2,406 & 2,246 & 160 & 15,407 & 37 & 35 & 2 & 1 \\
\hline 101 & Dugo Selo & 2,397 & 2,317 & 79 & 17,466 & 42 & 40 & 1 & 3 \\
\hline 102 & Daruvar & 2,385 & 2,239 & 146 & 11,633 & 28 & 26 & 2 & 3 \\
\hline 103 & Vrbovec & 2,327 & 2,331 & -4 & 14,797 & 34 & 34 & 0 & 3 \\
\hline 104 & Slatina & 2,307 & 2,117 & 190 & 13,686 & 32 & 29 & 3 & 3 \\
\hline 105 & Slavonski Brod & 2,245 & 1,870 & 375 & 59,141 & 133 & 111 & 22 & 5 \\
\hline 106 & Sinj & 2,222 & 2,110 & 112 & 24,826 & 55 & 52 & 3 & 1 \\
\hline 107 & Ozalj & 2,180 & 2,301 & -121 & 6,817 & 15 & 16 & -1 & 3 \\
\hline 108 & Ploče & 2,164 & 2,725 & -561 & 10,135 & 22 & 28 & -6 & 3 \\
\hline 109 & Nova Gradiška & 2,151 & 2,106 & 45 & 14,229 & 31 & 30 & 1 & 0 \\
\hline 110 & Otok(Vinkovci) & 2,124 & 1,788 & 336 & 6,343 & 13 & 11 & 2 & 0 \\
\hline 111 & Oroslavje & 2,072 & 1,983 & 89 & 6,138 & 13 & 12 & 1 & 2 \\
\hline 112 & Duga Resa & 2,039 & 2,193 & -154 & 11,180 & 23 & 25 & -2 & 4 \\
\hline 113 & Pleternica & 2,032 & 1,905 & 127 & 11,323 & 23 & 22 & 1 & 0 \\
\hline 114 & Čazma & 1,999 & 1,892 & 108 & 8,077 & 16 & 15 & 1 & 3 \\
\hline 115 & Sveti Ivan Zelina & 1,987 & 2,052 & -65 & 15,959 & 32 & 33 & -1 & 0 \\
\hline 116 & Donji Miholjac & 1,944 & 1,697 & 248 & 9,491 & 18 & 16 & 2 & 3 \\
\hline 117 & Trilj & 1,913 & 1,864 & 48 & 9,109 & 17 & 17 & 0 & 0 \\
\hline 118 & Zlatar & 1,850 & 1,255 & 594 & 6,096 & 11 & 8 & 4 & 4 \\
\hline 119 & Ivanec & 1,844 & 1,619 & 225 & 13,758 & 25 & 22 & 3 & 3 \\
\hline 120 & Pregrada & 1,829 & 1,832 & -4 & 6,594 & 12 & 12 & 0 & 4 \\
\hline 121 & Garešnica & 1,798 & 1,687 & 110 & 10,472 & 19 & 18 & 1 & 1 \\
\hline 122 & $\begin{array}{l}\text { Varaždinske } \\
\text { Toplice }\end{array}$ & 1,770 & 1,771 & -1 & 6,364 & 11 & 11 & 0 & 3 \\
\hline 123 & Valpovo & 1,742 & 1,741 & 1 & 11,563 & 20 & 20 & 0 & 2 \\
\hline 124 & Metković & 1,693 & 1,560 & 133 & 16,788 & 28 & 26 & 2 & 2 \\
\hline 125 & Mursko Središće & 1,593 & 1,487 & 106 & 6,307 & 10 & 9 & 1 & 1 \\
\hline 126 & Đakovo & 1,428 & 1,347 & 81 & 27,745 & 40 & 37 & 2 & 1 \\
\hline 127 & Kutjevo & 1,336 & 1,323 & 13 & 6,247 & 8 & 8 & 0 & 0 \\
\hline 128 & Novi Marof & 1,319 & 1,210 & 109 & 13,246 & 17 & 16 & 1 & 2 \\
\hline
\end{tabular}

Total (in million HRK) 
Table 3

Budget outturns of counties, 2014

Per capita ( in HRK)

Total (in million HRK)

\begin{tabular}{|c|c|c|c|c|c|c|c|c|c|}
\hline \multicolumn{5}{|c|}{ 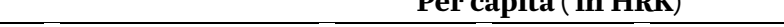 } & \multicolumn{5}{|c|}{ 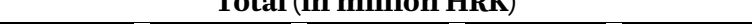 } \\
\hline & County & $\begin{array}{c}\text { Reve- } \\
\text { nues }\end{array}$ & $\begin{array}{l}\text { Expen- } \\
\text { ditures }\end{array}$ & $\begin{array}{c}\text { Surplus } \\
\text { or } \\
\text { deficit }\end{array}$ & $\begin{array}{l}\text { Popula- } \\
\text { tion }\end{array}$ & $\begin{array}{c}\text { Reve- } \\
\text { nues }\end{array}$ & $\begin{array}{l}\text { Expen- } \\
\text { ditures }\end{array}$ & $\begin{array}{c}\text { Surplus } \\
\text { or } \\
\text { deficit }\end{array}$ & $\begin{array}{c}\text { Level of } \\
\text { budget } \\
\text { trans- } \\
\text { parency }\end{array}$ \\
\hline 1 & $\begin{array}{l}\text { Virovitica- } \\
\text { Podravina }\end{array}$ & 1,436 & 1,363 & 72 & 84,836 & 122 & 116 & 6 & 3 \\
\hline 2 & Istria & 1,353 & 1,250 & 102 & 208,055 & 281 & 260 & 21 & 4 \\
\hline 3 & $\begin{array}{l}\text { Koprivnica- } \\
\text { Križevci }\end{array}$ & 1,269 & 1,223 & 46 & 115,584 & 147 & 141 & 5 & 4 \\
\hline 4 & Varaždin & 1,250 & 1,124 & 126 & 175,951 & 220 & 198 & 22 & 5 \\
\hline 5 & Lika-Senj & 1,191 & 1,179 & 12 & 50,927 & 61 & 60 & 1 & 4 \\
\hline 6 & Šibenik-Knin & 1,152 & 1,143 & 8 & 109,375 & 126 & 125 & 1 & 5 \\
\hline 7 & $\begin{array}{l}\text { Dubrovnik- } \\
\text { Neretva }\end{array}$ & 1,147 & 1,085 & 62 & 122,568 & 141 & 133 & 8 & 4 \\
\hline 8 & Karlovac & 1,147 & 1,155 & -9 & 128,899 & 148 & 149 & -1 & 4 \\
\hline 9 & Zadar & 1,126 & 1,065 & 62 & 170,017 & 191 & 181 & 10 & 5 \\
\hline 10 & Krapina-Zagorje & 1,100 & 1,096 & 4 & 132,892 & 146 & 146 & 1 & 5 \\
\hline 11 & Požega-Slavonija & 1,089 & 1,017 & 72 & 78,034 & 85 & 79 & 6 & 3 \\
\hline 12 & $\begin{array}{l}\text { Primorje-Gorski } \\
\text { Kotar }\end{array}$ & 1,058 & 1,002 & 57 & 296,195 & 313 & 297 & 17 & 4 \\
\hline 13 & Brod-Posavina & 1,033 & 1,029 & 4 & 158,575 & 164 & 163 & 1 & 5 \\
\hline 14 & Međimurje & 1,026 & 1,032 & -6 & 113,804 & 117 & 117 & -1 & 3 \\
\hline 15 & Bjelovar-Bilogora & 996 & 942 & 53 & 119,764 & 119 & 113 & 6 & 4 \\
\hline 16 & Vukovar-Srijem & 988 & 924 & 64 & 179,521 & 177 & 166 & 12 & 3 \\
\hline 17 & Osijek-Baranja & 958 & 937 & 21 & 305,032 & 292 & 286 & 7 & 4 \\
\hline 18 & Split-Dalmatia & 954 & 945 & 9 & 454,798 & 434 & 430 & 4 & 3 \\
\hline 19 & Sisak-Moslavina & 946 & 995 & -49 & 172,439 & 163 & 172 & -9 & 2 \\
\hline 20 & Zagreb & 912 & 886 & 26 & 317,606 & 290 & 282 & 8 & 4 \\
\hline
\end{tabular}

Source: The authors, based on the Ministry of Finance (2015) and CBS (2015); and Ott, Bronić and Petrušić (2015).

\section{LITERATURE}

Bajo, A. and Primorac, M., 2OI4. The debt and fiscal risks of local and regional self-government units in Croatia. Newsletter, No. 93.

CBS, 2015. Census of Population, Households and Dwellings 20II. Zagreb: Croatian Bureau of Statistics. IPF, 20I5. Local Budget Transparency. Zagreb: Institute of Public Finance.

Ministry of Finance, 2015. Local government units' budgets - archive (Croatian only). Zagreb: Ministry of Finance.

Ott, K., Bronić, M. and Petrušić, M., 2015. Budget transparency in Croatian counties, cities and municipalities (November 20I4 - March 2015). Newsletter, No. 97.

Zakon o proračunu (Budget Act), NN 87/o8, I36/I2 and I5/I5. Zagreb: Narodne novine. 